\title{
Chiral spirals from noncontinuous chiral symmetry: The Gross-Neveu model results
}

\author{
Toru Kojo \\ Department of Physics, University of Illinois, \\ 1110 West Green Street, Urbana, Illinois 61801, USA
}

(Dated: October 10, 2018)

\begin{abstract}
It is shown that the inhomogeneous chiral condensate in the Gross-Neveu (GN) model takes the chiral spiral form, even though the thermodynamic functional depends only on the chiral scalar density. It is the inhomogeneity of the chiral scalar condensate that drives the spatial modulations of the pseudoscalar one. The result has broader implications once we start to think of fundamental theories behind the effective models. In particular, some effective interactions - which may be omitted for descriptions of the homogeneous phases - can be dynamically enhanced due to the spatial modulations of the large mean fields. Implications for the four-dimensional counterparts of the GN model are discussed. In a quark matter context, proper forms of the effective models for the inhomogeneous phases are speculated, through considerations on the Fermi-Dirac sea coupling.
\end{abstract}




\section{INTRODUCTION}

Recently, phases of the inhomogeneous chiral condensates (IChCs) have attracted renewed attention in the quark matter context [1, 2]. A number of studies based on the NJL-type model [3, 4] as well as models with the infrared (IR) enhanced interactions [5, 6] have suggested that in some domain of moderate quark density the IChC phases are energetically more favored than the normal, chiral symmetric phase. In particular, the NJL-type model studies indicate that the phase of IChC may mask the usual first-order chiral phase transition line and its critical end point, and might change the conventional wisdom.

So far, most studies have been concentrated on the chiral condensates of the liquid crystal type, in which the condensates spatially modulate in one particular direction (say, the $z$ direction), while they are uniform in the other two directions. For the description of such phases, the model studies rely on the understanding of their two-dimensional counterparts: the Gross-Neveu (GN) model [7, 8] as a counterpart for the $\mathrm{NJL}_{4}$ model [3], the 't Hooft model $\left(\mathrm{QCD}_{2}\right)$ [9] for the confining model [5, 6], and the $\mathrm{NJL}_{2}$ model [10] for the extended $\mathrm{NJL}_{4}$ model with tensor 4-Fermi interactions [11]. In fact, the solutions of two-dimensional models can be naturally embedded into the four-dimensional mean field ansatz.

The inhomogeneous solutions for two-dimensional models are similar but not quite identical. The $\mathrm{QCD}_{2}$ and $\mathrm{NJL}_{2}$ models are known to have the chiral spiral ground states,

$$
\langle\bar{\psi} \psi\rangle_{2 \mathrm{D}}=\Delta \cos \left(2 p_{F} z\right), \quad\left\langle\bar{\psi} \mathrm{i} \gamma_{0} \gamma_{z} \psi\right\rangle_{2 \mathrm{D}}=\Delta \sin \left(2 p_{F} z\right), \quad\left(\gamma_{5}^{2 \mathrm{D}}=\gamma_{0} \gamma_{z}\right)
$$

which can be directly brought into its four-dimensional version by replacing $\left(\gamma_{0} \gamma_{z}\right)^{2 \mathrm{D}} \rightarrow$ $\left(\gamma_{0} \gamma_{z}\right)^{4 \mathrm{D}}$. (This solution should not be confused with the pionic chirals such as $\left\langle\bar{\psi} \mathrm{i} \gamma_{5} \psi\right\rangle_{4 \mathrm{D}}$ or $\left\langle\bar{\psi} \mathrm{i} \tau_{3} \gamma_{5} \psi\right\rangle_{4 \mathrm{D}}$ where $\gamma_{5}^{4 \mathrm{D}} \neq\left(\gamma_{0} \gamma_{z}\right)^{4 \mathrm{D}}[16]$.) Here $2 p_{F}$ appears because of the condensed pairs of comoving particle-holes near the Fermi surface. On the other hand, for the GN model, the spiral solution is usually not considered, because the 4-Fermi interaction takes the form $(\bar{\psi} \psi)^{2}$, so that its mean-field thermodynamic functional depends only on $\langle\bar{\psi} \psi\rangle$ but not on $\left\langle\bar{\psi} \mathrm{i} \gamma_{5} \psi\right\rangle$. Therefore, the above two classes of solutions are usually distinguished.

In this paper we explain how to understand differences between them by revisiting inhomogeneous solutions of the GN model [7]. To avoid confusion, we emphasize that we will not attempt to modify the analytic solution, which was shown to achieve the ground state [8]. On the other hand, there are physical implications which cannot be observed from the 
expression of the thermodynamic functional. In fact, not all condensates manifestly appear in the energy minimization procedure.

Using the analytically known fermion eigenstates, we compute condensates explicitly to show that the inhomogeneous condensate in the GN model actually takes the chiral spiral form. It is, however, not identical with those in $\mathrm{QCD}_{2}\left(\mathrm{NJL}_{2}\right)$. In the GN model, the net contribution to the chiral scalar density comes from the Dirac sea, while that for the chiral pseudoscalar density comes from the Fermi sea. This introduces disparities in amplitudes of two densities.

This chiral spiral solution in the GN model can be elevated to the $\mathrm{NJL}_{4}$ model. Like the GN model case, the corresponding chiral spiral-which is made of $\langle\bar{\psi} \psi\rangle_{4 \mathrm{D}}$ and $\left\langle\bar{\psi} \mathrm{i} \gamma_{0} \gamma_{z} \psi\right\rangle_{4 \mathrm{D}^{-}}$ cannot be observed from the thermodynamic functional in the $\mathrm{NJL}_{4}$ model, and it must be computed using the fermion bases in the scalar mean field of Ref. [3]. We will show the explicit mapping from two to four dimensions in another publication, but we think that the main features should already be clear from our two-dimensional analyses in this paper.

Actually, for the discussions of the QCD phase diagram, the derivation of the chiral spirals in the $\mathrm{NJL}_{4}$ model is not the end of the story. It leads to broader implications once we start to think of fundamental theories behind the effective models.

For the $\mathrm{NJL}_{4}$ model up to dimension-6 operators, in principle we should include all possible 4-Fermi interactions that are compatible with symmetries of QCD, although many of them can be discarded based on other set of arguments. For example, in vacuum, it does not matter whether or not we include tensor type interactions $\sim\left(\bar{\psi} \sigma_{\mu \nu} \psi\right)^{2}+\left(\bar{\psi} \mathrm{i} \gamma_{5} \sigma_{\mu \nu} \tau_{a} \psi\right)^{2}$, simply because the tensor mean field is zero, not because the coupling constant is small (there are no reasons why the coupling should be very small). The only important mean field comes from the scalar density, so that terms $\sim(\bar{\psi} \psi)^{2}+\left(\bar{\psi} \mathrm{i} \gamma_{5} \tau_{a} \psi\right)^{2}$ are enough to take into account relevant dynamical effects and, at the same time, maintain the chiral symmetry.

The situation is different for inhomogeneous phases. As explained above, the spatially modulating scalar density drives the spatial modulation of the tensor mean field $\left\langle\bar{\psi} \mathrm{i} \gamma_{0} \gamma_{z} \psi\right\rangle$ whose amplitude is comparable to the scalar one. In this case, the relevance of the tensortype interactions is dynamically enhanced, so we have to reanalyze the mean field solutions in the presence of such interactions. If the new mean fields turn out to generate another mean field, again we have to include the corresponding 4-Fermi interactions and reanalyze dynamics from the beginning. This procedure should be repeated until we exhaust all 
possible dynamically enhanced 4-Fermi interactions and mean fields. After that, we can pick out the effective models for the inhomogeneous phase.

This paper is organized as follows: In Sec. II, we review the inhomogeneous mean field solution for the GN model and reproduce a number of important results in Ref. [7]. We quickly summarize basics of the elliptic functions, to the extent necessary for converting the mathematical structure into physical terminology. In Sec. III, we calculate the expectation values of various operators- in particular, pseudoscalar density. By examining its relationship with the scalar density, we show that they form the chiral spirals with unequal amplitudes. In Sec. IV, we compare the chiral spirals in the GN model to $\mathrm{QCD}_{2}$ and the $\mathrm{NJL}_{2}$ model. Section $\mathrm{V}$ is devoted to summary.

In Sec. III and the appendixes, we add a number of supplementary materials for Ref. [7], because the descriptions in the original paper were rather dense and hard to access for nonexperts. We try to reduce the gaps between calculations in Ref. [7]. The relevant formula to be used can be found in a handbook for mathematics [12], and its derivation can be found in Ref. [13]. Throughout this paper, we use the convention $\left(x^{0}, x^{1}\right)=(t, x)$ and $g_{\mu \nu}=\operatorname{diag} .(1,-1)$.

\section{INHOMOGENEOUS MEAN FIELDS FOR THE GROSS-NEVEU MODEL}

The Gross-Neveu model with $N$ colors is

$$
\mathcal{L}=\bar{\psi} \mathrm{i} \not \partial \psi+\frac{G}{2 N}(\bar{\psi} \psi)^{2}
$$

where the sum over color indices are implicit. We consider $N \rightarrow \infty$ for the mean field considerations. Using the auxiliary field method, we have

$$
\mathcal{L}=\bar{\psi}[\mathrm{i} \not \partial-M(x)] \psi-\frac{N}{2 G} M^{2}(x)
$$

We are going to use the canonical approach to treat the system at finite density. The constraint will be treated in Sec. III, while in this section we just investigate properties of the eigenstates.

In Sec. II A, we first review the mean field Ansatz and some properties of the elliptic functions. In Sec. II B, we summarize properties of the fermion eigenstates such as relations between the energy and quasimomentum. The density of states is given in Sec. IIC. How 
to map the UV cutoff from the homogeneous to the inhomogeneous phase is explained in Sec. IID,

\section{A. Field equations}

We first analyze the Dirac equation. The field equation is

$$
\left[-\mathrm{i} \gamma^{5} \partial_{1}+M(x) \gamma^{0}\right] \psi=\omega \psi
$$

To proceed further, we choose the $\gamma$ matrices and spinor bases as

$$
\gamma^{0}=-\sigma_{1}, \quad \gamma^{1}=\mathrm{i} \sigma_{3}, \quad \gamma^{5}=\gamma^{0} \gamma^{1}=-\sigma_{2}, \quad \psi_{\omega}(x)=\left[\begin{array}{c}
\varphi_{\omega}(x) \\
\chi_{\omega}(x)
\end{array}\right]
$$

Then the field equation takes the form

$$
\left[\partial_{1}-M(x)\right] \chi=\omega \varphi, \quad\left[-\partial_{1}-M(x)\right] \varphi=\omega \chi
$$

The reason to take the above bases is that the Dirac equation with a mean field can be brought into the Lame form, whose analytic properties have been investigated (Refs. [14] and [15] are very useful). From this set of equations, we can find

$$
\left[-\frac{\partial^{2}}{\partial x^{2}}-\frac{\partial M}{\partial x}+M^{2}\right] \varphi=\omega^{2} \varphi, \quad\left[-\frac{\partial^{2}}{\partial x^{2}}+\frac{\partial M}{\partial x}+M^{2}\right] \chi=\omega^{2} \chi .
$$

Now we consider the ansatz proposed by Thies [7]. Its form is

$$
M(x)=\mathcal{A} \lambda \frac{\operatorname{sn}(\mathcal{A} x \mid \lambda) \operatorname{cn}(\mathcal{A} x \mid \lambda)}{\operatorname{dn}(\mathcal{A} x \mid \lambda)} \equiv \mathcal{A} \mathcal{M}(\xi \mid \lambda), \quad \xi=\mathcal{A} x,
$$

where sn, cn, and dn are Jacobi's elliptic functions with the elliptic modulus $\lambda$. $\mathcal{A}$ and $\lambda$ are variational parameters. To get feelings about the ansatz, let us briefly look at basic properties of the elliptic functions:

(i) The elliptic functions interpolate the trigonometric functions and hyperbolic functions through the elliptic parameter $\lambda[17]$. In the $\lambda \rightarrow 0$ limit,

$$
\operatorname{sn}(\xi \mid 0)=\sin \xi, \quad \operatorname{cn}(\xi \mid 0)=\cos \xi, \quad \operatorname{dn}(\xi \mid 0)=1
$$

and in the $\lambda \rightarrow 1$ limit,

$$
\operatorname{sn}(\xi \mid 1)=\tanh \xi, \quad \operatorname{cn}(\xi \mid 1)=\operatorname{sech} \xi, \quad \operatorname{dn}(\xi \mid 1)=\operatorname{sech} \xi
$$




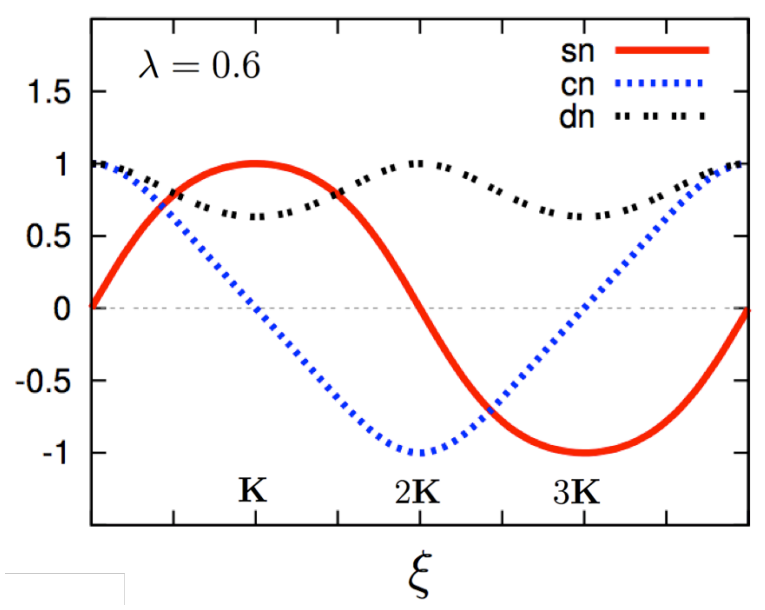

FIG. 1: The Jacobi elliptic functions, $\operatorname{sn}(\xi \mid \lambda), \operatorname{cn}(\xi \mid \lambda)$, and $\operatorname{dn}(\xi \mid \lambda)$ at $\lambda=0.6 . \mathbf{K}(\lambda)$ is the quarter period.

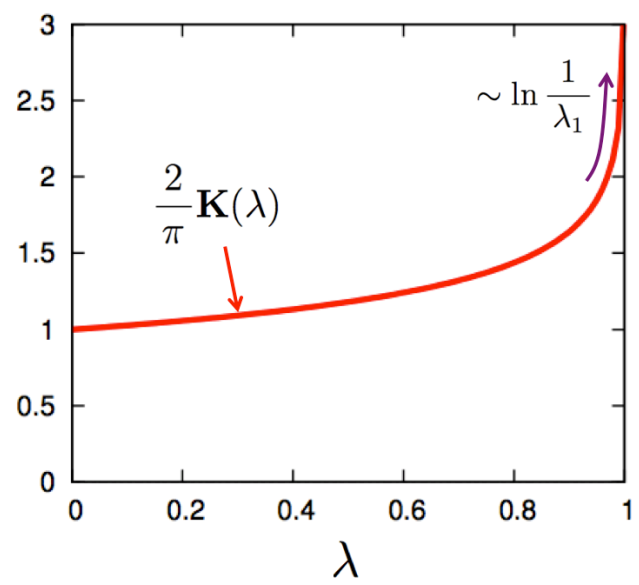

FIG. 2: The Jacobi complete elliptic function of the first kind $\mathbf{K}(\lambda)$ (normalized by $\pi / 2$ ). The period grows logarithmically as $\lambda$ approaches 1 , which corresponds to the dilute limit of the fermion density.

with which

$$
\mathcal{M}(\xi \mid \lambda \rightarrow 0) \sim \frac{\lambda}{2} \sin (2 \xi), \quad \mathcal{M}(\xi \mid \lambda \rightarrow 1) \sim \pm \tanh (\xi)
$$

The $\lambda \rightarrow 0$ limit corresponds to the density wave solution at high density, and the $\lambda \rightarrow 1$ limit corresponds to solitonic solutions at low density. As an example, in Fig.1 we plot these functions for $\lambda=0.6$. This asymptotic behavior motivates us to use the ansatz interpolating these two solutions which are known to minimize the thermodynamic potential.

(ii) As with trigonometric functions, there are simple square relations,

$$
\operatorname{sn}^{2}(\xi \mid \lambda)+\operatorname{cn}^{2}(\xi \mid \lambda)=1, \quad \operatorname{dn}^{2}(\xi \mid \lambda)=1-\lambda \operatorname{sn}^{2}(\xi \mid \lambda)
$$

(iii) As with trigonometric functions, we can define the quarter period. It is given by the 
Jacobi complete elliptic integral of the first kind [18],

$$
\mathbf{K}(\lambda)=\int_{0}^{\pi / 2} \frac{\mathrm{d} \theta}{\sqrt{1-\lambda \sin ^{2} \theta}} \rightarrow\left\{\begin{array}{cc}
\pi / 2 & (\lambda \rightarrow 0) \\
\frac{1}{2} \ln \frac{16}{\lambda_{1}} & (\lambda \rightarrow 1)
\end{array}\right.
$$

where $\lambda_{1} \equiv 1-\lambda$ is called the complementary modulus of $\lambda$. As the name suggests, $\operatorname{sn}(\xi+$ $4 \mathbf{K})=\operatorname{sn}(\xi), \operatorname{cn}(\xi+4 \mathbf{K})=\operatorname{cn}(\xi), \cdots$ are satisfied for any values of $\lambda$. The changes for the quarter period are more nontrivial, and there are formulas. [19] (Below, we sometimes omit $\lambda$ as long as it does not bring any confusion.)

$$
\operatorname{sn}(\xi \pm \mathbf{K})= \pm \frac{\operatorname{cn}(\xi)}{\operatorname{dn}(\xi)}, \quad \operatorname{cn}(\xi \pm \mathbf{K})=\mp \lambda_{1}^{1 / 2} \frac{\operatorname{sn}(\xi)}{\operatorname{dn}(\xi)}, \quad \operatorname{dn}(\xi \pm \mathbf{K})=\lambda_{1}^{1 / 2} \frac{1}{\operatorname{dn}(\xi)}
$$

with which we can show

$$
\mathcal{M}(\xi)=-\mathcal{M}(\xi \pm \mathbf{K})=\mathcal{M}(\xi \pm 2 \mathbf{K})
$$

The first equality can be used to cast the equation for $\chi$ into the same form as for $\varphi$. For later convenience, we rescale variables as

$$
(\varphi(x), \chi(x)) \equiv\left(\tilde{\varphi}\left(\mathcal{A}^{-1} x\right), \tilde{\chi}\left(\mathcal{A}^{-1} x\right)\right)=(\tilde{\varphi}(\xi), \tilde{\chi}(\xi)), \quad \tilde{\omega} \equiv \omega / \mathcal{A}
$$

and using Eq.(15), we can rewrite Eq.(7) as

$$
\left[-\frac{\partial^{2}}{\partial \xi^{2}}-\frac{\partial \mathcal{M}(\xi)}{\partial \xi}+\mathcal{M}^{2}(\xi)\right] f(\xi)=\tilde{\omega}^{2} f(\xi), \quad f(\xi)=(\tilde{\varphi}(\xi), \tilde{\chi}(\xi \pm \mathbf{K})) .
$$

Note that $\tilde{\varphi}(\xi)$ and $\tilde{\chi}(\xi \pm \mathbf{K})$ satisfy the same equations, so one of the solutions can be related to the other by shifting the coordinate by $\mathbf{K}$, modulo the relative phase factor.

(iv) The derivatives of the elliptic functions are given by [20]

$$
\frac{\mathrm{d} \operatorname{sn}(\xi)}{\mathrm{d} \xi}=\operatorname{cn}(\xi) \operatorname{dn}(\xi), \quad \frac{\mathrm{d} \operatorname{cn}(\xi)}{\mathrm{d} \xi}=-\operatorname{sn}(\xi) \operatorname{dn}(\xi), \quad \frac{\mathrm{d} \mathrm{dn}(\xi)}{\mathrm{d} \xi}=-\lambda \operatorname{sn}(\xi) \operatorname{cn}(\xi),
$$

with which one gets

$$
\frac{\mathrm{d} \mathcal{M}(\xi)}{\mathrm{d} \xi}=-2 \lambda \operatorname{sn}^{2}(\xi)+\lambda+\mathcal{M}^{2}
$$

Finally, we arrive at the Lame form of the eigenvalue equation:

$$
\left[-\frac{\partial^{2}}{\partial \xi^{2}}+2 \lambda \operatorname{sn}^{2}(\xi)\right] f(\xi)=\left(\tilde{\omega}^{2}+\lambda\right) f(\xi)
$$

The number 2 in front of $\operatorname{sn}^{2}(\xi)$ is the special case of $l(l+1)$. For given $l$, the equation has $2 l$ gaps in the energy spectra $[13$. 


\section{B. Eigenvalues and eigenfunctions}

To study the eigenstates, let us first note that the period of the potential is $2 \mathbf{K}(\lambda)$. Therefore the eigenfunction must take the Bloch form:

$$
\Psi_{Q}(\xi)=v_{Q}(\xi) \mathrm{e}^{\mathrm{i} \tilde{Q} \xi}, \quad v_{Q}(\xi)=v_{Q}(\xi+2 \mathbf{K}),
$$

where $\tilde{Q}=Q / \mathcal{A}$ is the (dimensionless) quasimomentum which is a real, continuous variable. On the other hand, the Fourier modes for $v_{Q}(\xi)$ can take only discrete values, $n \pi / \mathbf{K}$, where $n$ is an integer. Note also that the equation is the second-order differential one, and its kernel is real, so that we have a pair of solutions $(\tilde{\varphi}, \tilde{\chi})$, and $\left(\tilde{\varphi}^{*}, \tilde{\chi}^{*}\right)$.

Explicitly, the solution of the Lame equation for $l=1$ is given by [13, 14]

$$
f_{\alpha}(\xi \mid \lambda)=\frac{\theta_{1}\left(u_{\xi+\alpha}, q \mid \lambda\right)}{\theta_{4}\left(u_{\xi}, q \mid \lambda\right)} \mathrm{e}^{\xi Z(\alpha \mid \lambda)}, \quad f_{\alpha}^{*}(\xi \mid \lambda)=\frac{\theta_{1}^{*}\left(u_{\xi+\alpha}, q \mid \lambda\right)}{\theta_{4}\left(u_{\xi}, q \mid \lambda\right)} \mathrm{e}^{\xi Z^{*}(\alpha \mid \lambda)},
$$

where $\theta_{a}\left(u_{\xi}, q\right)$ and $Z(\alpha \mid \lambda)$ are Jacobi elliptic theta and zeta functions with the modulus $\lambda$, and the variables $u_{\xi}$ and $q$ (called "nome") are

$$
u_{\xi} \equiv \frac{\pi \xi}{2 \mathbf{K}(\lambda)} \rightarrow\left\{\begin{array}{cc}
\xi & (\lambda \rightarrow 0) \\
\frac{\pi \xi}{\ln \frac{16}{\lambda_{1}}} & (\lambda \rightarrow 1)
\end{array}, \quad q \equiv \mathrm{e}^{-\pi \mathbf{K}^{\prime} / \mathbf{K}} \rightarrow\left\{\begin{array}{cc}
\frac{\lambda}{16} & (\lambda \rightarrow 0) \\
\mathrm{e}^{-\frac{\pi^{2}}{\ln \left(16 / \lambda_{1}\right)}} & (\lambda \rightarrow 1)
\end{array}\right.\right.
$$

where $\mathbf{K}^{\prime}=\mathbf{K}^{\prime}(\lambda)=\mathbf{K}\left(\lambda_{1}\right)$. Below we convert the abstract expressions into physical notions.

(i) The parameter $\alpha$ is directly related to the energy spectra by the following relation:

$$
\operatorname{dn}^{2}(\alpha \mid \lambda)=\tilde{\omega}^{2} .
$$

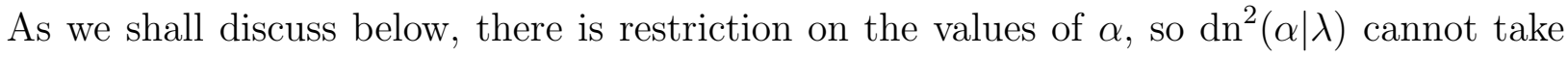
arbitrary values. Accordingly, there are forbidden regions for $\tilde{\omega}$ which appear as the energy gaps in the spectra.

(ii) We can identify the Bloch periodic function and quasimomentum as

$$
v_{Q}(\xi) \equiv \frac{\theta_{1}\left(u_{\xi+\alpha}, q \mid \lambda\right)}{\theta_{4}\left(u_{\xi}, q \mid \lambda\right)} \exp \frac{-\mathrm{i} \pi \xi}{2 \mathbf{K}}, \quad \tilde{Q} \equiv-\mathrm{i} Z(\alpha \mid \lambda)+\frac{\pi}{2 \mathbf{K}},
$$

which satisfy the condition $v_{Q}=v_{Q+2 \mathbf{K}}$. To understand this decomposition, we note that the series expansions for Jacobi ellliptic theta functions are [21]

$$
\begin{aligned}
& \theta_{1}(u, q)=2 q^{1 / 4} \sum_{n=0}^{\infty}(-1)^{n} q^{n(n+1)} \sin (2 n+1) u, \quad \theta_{1}(u)=\theta_{1}^{*}\left(u^{*}\right) \\
& \theta_{4}(u, q)=1+2 \sum_{n=1}^{\infty}(-1)^{n} q^{n^{2}} \cos 2 n u
\end{aligned}
$$




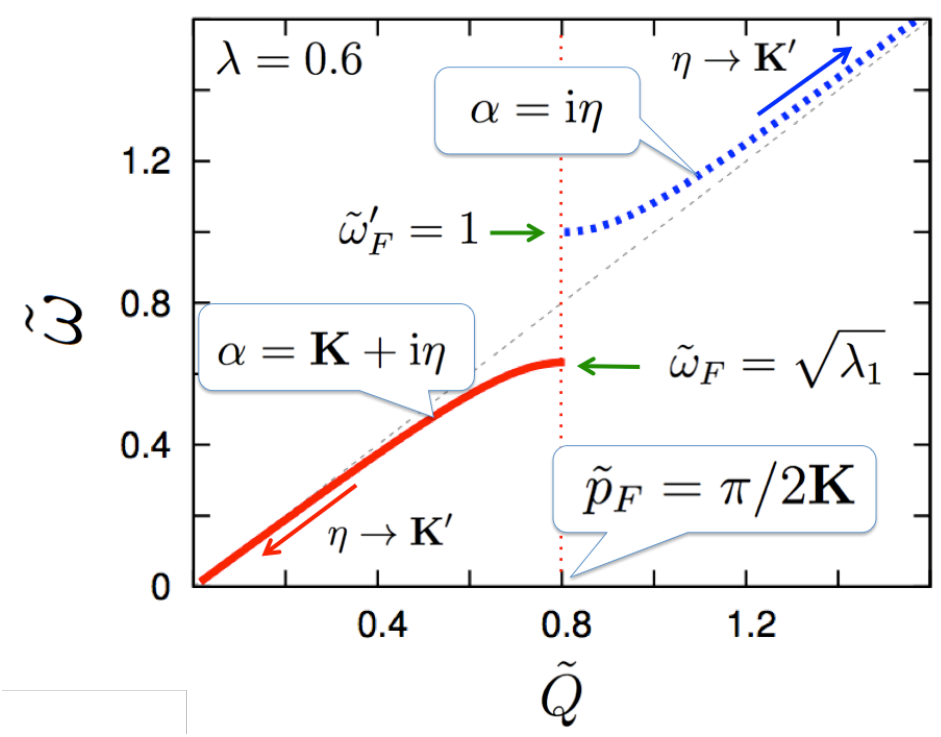

FIG. 3: The dispersion relation between the energy $\tilde{\omega}$ and quasimomentum $\tilde{Q}$ (normalized by $\mathcal{A}$ ). $\lambda$ is chosen to be 0.6 . The gap is opened at the quasimomentum $\pi / 2 \mathbf{K}(\eta=0)$, which should be assigned as $\tilde{p}_{F}$. The energy at the band edge is $\tilde{\omega}_{F}=\sqrt{\lambda_{1}}\left(\tilde{\omega}_{F}^{\prime}=1\right)$ for the first (second) energy branch. The plot is symmetric with respect to $\tilde{\omega} \rightarrow-\tilde{\omega}$, and the Dirac sea also has an energy gap of the same size.

from which we can verify

$$
\theta_{1}\left(u_{\xi}+u_{2 \mathbf{K}}\right)=\theta_{1}\left(u_{\xi}+\pi\right)=-\theta_{1}\left(u_{\xi}\right), \quad \theta_{4}\left(u_{\xi}+u_{2 \mathbf{K}}\right)=\theta_{4}\left(u_{\xi}\right)
$$

The sign flipping in the first relation is the reason why we had to include $\mathrm{e}^{-\mathrm{i} \pi \xi / 2 \mathbf{K}}$ in $v_{Q}$.

(iii) The quasimomentum $\tilde{Q}$ must be a real variable, so $Z(\alpha \mid \lambda)$ must be purely imaginary. This constrains the value of $\alpha$. The series expansion of the zeta function takes the form [22]

$$
Z(\alpha \mid \lambda)=\frac{2 \pi}{\mathbf{K}} \sum_{n=1}^{\infty} \frac{q^{n}}{1-q^{2 n}} \sin \left(\frac{n \pi}{\mathbf{K}} \alpha\right),
$$

which has periodicity $2 \mathbf{K}$ in $\alpha$. Thus $\alpha$ must take the form $[23]$

$$
\alpha=\mathrm{i} \eta, \quad \alpha=\mathbf{K}+\mathrm{i} \eta, \quad \eta \in\left[0, \mathbf{K}^{\prime}\right] .
$$

Note that at $\eta=0$ we have $Z(0)=Z(\mathbf{K})=0$, meaning that the quasimomenta of two branches coincide. This is the momentum where the energy gap appears; see Fig. 3. As we will see later, to minimize the energy of the system, the quasimomentum at the gap should be taken to be $p_{F} / \mathcal{A}$,

$$
\tilde{Q}=\pi / 2 \mathbf{K}=p_{F} / \mathcal{A}, \quad(\eta=0)
$$


so that the first positive energy branch is perfectly filled while the second positive energy branch is empty. This determines $\mathcal{A}$ as a function of $\lambda$.

(iv) The energy branches are determined as follows. We first examine $\alpha=\mathrm{i} \eta$ (second energy branch). Using Jacobi's imaginary transformation [24],

$$
\operatorname{dn}(i \eta \mid \lambda)=\frac{\operatorname{dn}\left(\eta \mid \lambda_{1}\right)}{\operatorname{cn}\left(\eta \mid \lambda_{1}\right)}
$$

(note that the modulus on the rhs is $\lambda_{1}=1-\lambda$ ), we arrive at an equation for the second energy branch,

$$
\tilde{\omega}^{2}=\frac{\operatorname{dn}^{2}\left(\eta \mid \lambda_{1}\right)}{\operatorname{cn}^{2}\left(\eta \mid \lambda_{1}\right)}=\lambda_{1}+\frac{1-\lambda_{1}}{\operatorname{cn}^{2}\left(\eta \mid \lambda_{1}\right)} \geq 1 \equiv \tilde{\omega}_{F}^{\prime 2} .
$$

Next, we examine $\alpha=\mathbf{K}+$ i $\eta$ (first energy branch). Using the relation for the quarter period (14) and then Jacobi's imaginary transformation (31), we get

$$
\operatorname{dn}(\mathbf{K}+\mathrm{i} \eta \mid \lambda)=\frac{\lambda_{1}^{1 / 2}}{\operatorname{dn}(\mathrm{i} \eta \mid \lambda)}=\lambda_{1}^{1 / 2} \frac{\operatorname{cn}\left(\eta \mid \lambda_{1}\right)}{\operatorname{dn}\left(\eta \mid \lambda_{1}\right)},
$$

and then we arrive at an equation for the first energy branch,

$$
\tilde{\omega}^{2}=\lambda_{1} \frac{\operatorname{cn}^{2}\left(\eta \mid \lambda_{1}\right)}{\operatorname{dn}^{2}\left(\eta \mid \lambda_{1}\right)}=1-\frac{1-\lambda_{1}}{\operatorname{dn}^{2}\left(\eta \mid \lambda_{1}\right)} \leq \lambda_{1} \equiv \tilde{\omega}_{F}^{2} .
$$

Therefore we find the energy gap between edges of the two branches, $\tilde{\omega}_{F}^{2}=\lambda_{1}$ and $\tilde{\omega}_{F}^{\prime 2}=1$. Because the relation is given for $\tilde{\omega}^{2}$, we have the gaps not only near the Fermi points but also in the Dirac sea. The energies as functions of quasimomenta are plotted in Fig. 3.

(v) In the following calculations, we assign eigenfunctions for $\tilde{\varphi}$ and $\tilde{\varphi}^{*}$ as

$$
\tilde{\varphi}_{\omega}(\xi)=\mathcal{N} \frac{\theta_{1}\left(u_{\xi+\alpha}, q \mid \lambda\right)}{\theta_{4}\left(u_{\xi}, q \mid \lambda\right)} \mathrm{e}^{\xi Z(\alpha \mid \lambda)}, \quad \tilde{\varphi}_{\omega}^{*}(\xi)=\mathcal{N}^{*} \frac{\theta_{1}\left(u_{\xi+\alpha^{*}}, q \mid \lambda\right)}{\theta_{4}\left(u_{\xi}, q \mid \lambda\right)} \mathrm{e}^{-\xi Z(\alpha \mid \lambda)}
$$

where $\mathcal{N}$ is the normalization factor. The relation between these two functions is like that between $\mathrm{e}^{\mathrm{i} k x}$ and $\mathrm{e}^{-\mathrm{i} k x}$ in a free fermion theory. On the other hand, they are related to $\chi_{\omega}$ and $\chi_{\omega}^{*}$ through Eq.(6). Actually the results in this paper do not require the expression of the relative phase factor. But we give the result for completeness, and it is given by (for the derivation, see Appendix.B]

$$
\tilde{\chi}_{\omega}(\xi)=\mathrm{e}^{\mathrm{i} \Phi(\omega)} \tilde{\varphi}_{\omega}(\xi-\mathbf{K}), \quad \mathrm{e}^{\mathrm{i} \Phi(\omega)} \equiv \operatorname{sgn}(\tilde{\omega}) \mathrm{e}^{\mathbf{K} Z(\alpha)},
$$

where $\Phi$ is real and the function $\chi(\xi)$ is proportional to $\varphi(\xi-\mathbf{K})$, as stated earlier. Note also that the phase factor changes the sign for $\omega \rightarrow-\omega$, as we can see from Eq. (6). 
(vi) Finally we fix the normalization. Since the wave function has periodicity of $2 \mathbf{K}$, the normalization condition is

$$
1=\frac{1}{2 \mathbf{K}} \int_{0}^{2 \mathbf{K}} \mathrm{d} \xi\left(|\tilde{\varphi}(\xi)|^{2}+|\tilde{\chi}(\xi)|^{2}\right)=\frac{1}{2 \mathbf{K}} \int_{0}^{2 \mathbf{K}} \mathrm{d} \xi\left(|\tilde{\varphi}(\xi)|^{2}+|\tilde{\varphi}(\xi-\mathbf{K})|^{2}\right) .
$$

We will give the explicit form of $\mathcal{N}$ in Appendix.C. Instead, here we give only the normalized expression for $|\tilde{\varphi}|^{2}$,

$$
\left|\tilde{\varphi}_{\omega}(\xi)\right|^{2}=\frac{1}{2}\left[1-\frac{\mathrm{dn}^{2}(\xi)-\mathbf{E} / \mathbf{K}}{\tilde{\omega}^{2}-\mathbf{E} / \mathbf{K}}\right],
$$

where $\mathbf{E}=\mathbf{E}(\lambda)$ is the complete elliptic integral of the second kind [25],

$$
\mathbf{E}(\lambda)=\int_{0}^{\pi / 2} \mathrm{~d} \theta \sqrt{1-\lambda \sin ^{2} \theta}=\int_{0}^{\mathbf{K}} \mathrm{d} \xi \mathrm{dn}^{2}(\xi \mid \lambda) \rightarrow\left\{\begin{array}{cc}
\pi / 2 & (\lambda \rightarrow 0) \\
1 & (\lambda \rightarrow 1)
\end{array}\right.
$$

Note that $\int_{0}^{2 \mathbf{K}} \mathrm{d} \xi\left[\mathrm{dn}^{2}(\xi)-\mathbf{E} / \mathbf{K}\right]=0$, so the spatial average of $|\tilde{\varphi}|^{2}$ is saturated by the first term in Eq. 38 .

\section{Density of states}

In various computations we will use the density of states. We take a derivative for the quasimomentum,

$$
\mathrm{d} \tilde{Q}=\frac{\mathrm{d} \tilde{Q}}{\mathrm{~d} \tilde{\omega}} \mathrm{d} \tilde{\omega}=\frac{\mathrm{d} \tilde{Q}}{\mathrm{~d} \alpha} \frac{\mathrm{d} \alpha}{\mathrm{d} \tilde{\omega}} \mathrm{d} \tilde{\omega}, \quad \mathcal{D}(\omega) \equiv\left|\frac{\mathrm{d} \tilde{Q}}{\mathrm{~d} \tilde{\omega}}\right|
$$

The $\alpha$ and $\tilde{\omega}$ are related through the relation (24). Let us first note that

$$
\frac{\mathrm{d}}{\mathrm{d} \tilde{\omega}} \operatorname{dn}(\alpha)=\frac{\mathrm{d}}{\mathrm{d} \tilde{\omega}} \tilde{\omega} \quad \leftrightarrow \quad \frac{\mathrm{d} \alpha}{\mathrm{d} \tilde{\omega}}=\frac{1}{\lambda} \frac{1}{\operatorname{sn}(\alpha) \operatorname{cn}(\alpha)}
$$

where either $\operatorname{sn}(\alpha)$ or $\operatorname{cn}(\alpha)$ becomes purely imaginary. Next we deal with $\mathrm{d} \tilde{Q} / \mathrm{d} \alpha$. Taking a derivative of the dispersion relation (see Appendix.D), we find

$$
\frac{\mathrm{d} \tilde{Q}}{\mathrm{~d} \alpha}=-\mathrm{i} \frac{\mathrm{d} Z(\alpha)}{\mathrm{d} \alpha}=-\mathrm{i}\left(\operatorname{dn}^{2}(\alpha)-\frac{\mathbf{E}}{\mathbf{K}}\right) .
$$

Assembling all these pieces, we arrive at

$$
\mathcal{D}(\omega)=\mp \frac{\tilde{\omega}^{2}-\mathbf{E} / \mathbf{K}}{\sqrt{\left(\tilde{\omega}^{2}-1\right)\left(\tilde{\omega}^{2}-\lambda_{1}\right)}} . \quad\left(- \text { for } 0 \leq \tilde{\omega}^{2} \leq \lambda_{1}, \quad+\text { for } 1 \leq \tilde{\omega}^{2}\right)
$$

Note that $\tilde{\omega}^{2}=\mathbf{E} / \mathbf{K}$ is in the forbidden region between the first and second energy branches. In fact there is an inequality $\lambda_{1} \leq \mathbf{E} / \mathbf{K} \leq 1$ which can be derived by noting that $\lambda_{1} \leq \operatorname{dn}^{2} x \leq$ 1 and $\int_{0}^{\mathbf{K}} \mathrm{d} x \operatorname{dn}^{2}(x)=\mathbf{E}$. Note that the density of states is enhanced near the band edges. 


\section{Mapping of the UV cutoff}

Finally, we relate the UV cutoff. Details will be given in Appendix E, but we will give the outline here. First, we notice that $\operatorname{dn}(\alpha)$ for $\alpha=\mathrm{i} \eta$ approaches $+\infty$ as $\alpha \rightarrow \mathrm{i} \mathbf{K}^{\prime}(\lambda)$. Introducing an infinitesimal quantity $\epsilon$, our energy cutoff for the inhomogenous phase, $\omega_{\Lambda}$, can be expressed as

$$
\omega_{\Lambda} / \mathcal{A}=\operatorname{dn}\left(\mathrm{i}\left(\mathbf{K}^{\prime}-\epsilon\right) \mid \lambda\right)
$$

On the other hand, the number of states in the Dirac sea is limited by $k=\Lambda$. Using the expression for the quasimomentum, we can write the momentum cutoff as

$$
\Lambda / \mathcal{A}=-\mathrm{i} Z\left(\mathrm{i}\left(\mathbf{K}^{\prime}-\epsilon\right) \mid \lambda\right)+\frac{\pi}{2 \mathbf{K}(\lambda)}
$$

Expanding these equations by $\epsilon$, we can eliminate $\epsilon$ and then relate the momentum cutoff to the energy cutoff,

$$
\omega_{\Lambda}^{2}=\Lambda^{2}+\mathcal{A}^{2}\left[-\lambda+2\left(1-\frac{\mathbf{E}}{\mathbf{K}}\right)\right]+O\left(\Lambda^{-2}\right) .
$$

The $O(1)$ terms must be kept during the following calculations.

\section{EXPECTATION VALUES}

Now we have all the ingredients to compute various quantities. We first write down expressions for the fermion number density and energy density, and then determine the variational parameters $\mathcal{A}$ and $\lambda$ as functions of average density or $p_{F}$. After that we compute the spatial modulations of various density operators: fermion number, energy, and scalar and pseudoscalar density. At the end of this section, in Sec. IIIE, we examine the high and low density limits of various quantities to get qualitative insights.

Using the bases found in the previous section, the fermion field operator can be written in terms of the creation and annihilation operators,

$$
\hat{\psi}_{c}(x)=\int \frac{\mathrm{d} \omega}{2 \pi} \sqrt{\mathcal{D}(\omega)} \sum_{j=1,2} u_{\omega}^{j}(\xi)\left[\theta(\omega) \mathrm{e}^{-\mathrm{i} \omega t} \hat{a}_{c}(\omega, j)+\theta(-\omega) \mathrm{e}^{\mathrm{i} \omega t} \hat{b}_{c}^{\dagger}(\omega, j)\right]
$$

where $\hat{a}$ and $\hat{b}$ are annihilation operators for particles and antiparticles, and $c$ is used for color indices. $\mathcal{D}(\omega)$ is the density of states, and we took the normalization of the creation and annihilation operators to satisfy $\left\{a_{c}(\omega, j), a_{c^{\prime}}^{\dagger}\left(\omega^{\prime}, j^{\prime}\right)\right\}=2 \pi \delta\left(\omega-\omega^{\prime}\right) \delta_{j j^{\prime}} \delta_{c c^{\prime}}$. [This normalization 
requires $\sqrt{\mathcal{D}}$ in Eq. 47 .] The wave functions giving energy $\omega$ are

$$
u_{\omega}^{1}(\xi)=\left[\begin{array}{c}
\tilde{\varphi}_{\omega}(\xi) \\
\tilde{\chi}_{\omega}(\xi)
\end{array}\right], \quad u_{\omega}^{2}(\xi)=\left[\begin{array}{c}
\tilde{\varphi}_{\omega}^{*}(\xi) \\
\tilde{\chi}_{\omega}^{*}(\xi)
\end{array}\right], \quad \tilde{\varphi}_{\omega}=\tilde{\varphi}_{-\omega}, \quad \tilde{\chi}_{\omega}=-\tilde{\chi}_{-\omega}
$$

where for the convention $\tilde{\varphi}_{\omega}=\tilde{\varphi}_{-\omega}$, the relative sign in $\tilde{\chi}_{\omega}$ for the positive and negative energy accompanies $(-1)$.

\section{A. Fermion number: Determination of $\mathcal{A}$}

The fermion number density is given by

$$
\left\langle\psi^{\dagger} \psi\right\rangle=\int \frac{\mathrm{d} \omega}{2 \pi} \mathcal{D}(\omega) \sum_{j=1,2}\left|u_{\omega}^{j}(\xi)\right|^{2}\left\langle\theta(\omega) \hat{a}^{\dagger}(\omega, j) \hat{a}(\omega, j)+\theta(-\omega)\left[1-\hat{b}^{\dagger}(\omega, j) \hat{b}(\omega, j)\right]\right\rangle,
$$

where the sum over color indices is implicit. Considering the fermion number constraint and the fact that the Dirac sea does not contain any antiparticles, we may require

$$
\left\langle\hat{a}^{\dagger}(\omega, j) \hat{a}(\omega, j)\right\rangle=N \theta\left(\epsilon_{F}-\omega\right), \quad \theta(-\omega)\left\langle\hat{b}^{\dagger}(\omega, j) \hat{b}(\omega, j)\right\rangle=0
$$

where $\epsilon_{F}$ is the Fermi energy which will be fixed below. We arrive at

$$
\begin{aligned}
\left\langle\psi^{\dagger} \psi(x)\right\rangle & =2 N\left(\int_{0}^{\epsilon_{F}}+\int_{-\omega_{\Lambda}}^{0}\right) \frac{\mathrm{d} \omega}{2 \pi} \mathcal{D}(\omega)\left(\left|\tilde{\varphi}_{\omega}(\xi)\right|^{2}+\left|\tilde{\chi}_{\omega}(\xi)\right|^{2}\right) \\
& =N\left(\int_{0}^{\epsilon_{F}}+\int_{-\omega_{\Lambda}}^{0}\right) \frac{\mathrm{d} \omega}{2 \pi} \mathcal{D}(\omega)\left[2-\frac{\mathcal{F}(\xi)}{\omega^{2} / \mathcal{A}^{2}-\mathbf{E} / \mathbf{K}}\right] .
\end{aligned}
$$

where we have defined

$$
\mathcal{F}(\xi) \equiv \operatorname{dn}^{2}(\xi)+\operatorname{dn}^{2}(\xi-\mathbf{K})-2 \mathbf{E} / \mathbf{K}
$$

whose spatial average over the period $2 \mathbf{K}$ is zero. Here we took into account the particles which fill the Dirac sea. The average part in the Dirac sea will be eliminated by the vacuum subtraction, while the spatial modulation is not and requires some care.

(i) Average density: We first compute the constant part. In order to minimize the energy, the particle should fill the first valence band, leaving the upper energy branch empty. In Appendix.F, we will show that locating the Fermi surface at the gapped points indeed reduces the energy density. Therefore, in the following, we set $\epsilon_{F}=\omega_{F}=\sqrt{\lambda_{1}} \mathcal{A}$. Next, notice that we impose the UV cutoff on momenta, so the size of phase space in the Dirac sea 
is kept fixed. Therefore, the Dirac sea contribution to the femion number density is common for all phases. Thus, subtracting the Dirac sea contribution, we demand that

$$
\frac{\left\langle\psi^{\dagger} \psi(x)\right\rangle_{\text {ave. }}^{R}}{N}=\int_{0}^{\omega_{F}=\sqrt{\lambda_{1}} \mathcal{A}} \frac{\mathrm{d} \omega}{\pi} \frac{\mathcal{A}^{2} \mathbf{E} / \mathbf{K}-\omega^{2}}{\sqrt{\left(\omega^{2}-\mathcal{A}^{2}\right)\left(\omega^{2}-\lambda_{1} \mathcal{A}^{2}\right)}}=\frac{p_{F}}{\pi} .
$$

Taking the variable $\omega=\sqrt{\lambda_{1}} \mathcal{A} t$, the integral can be expressed as

$$
\frac{\mathcal{A}}{\pi} \int_{0}^{1} \mathrm{~d} t\left[\sqrt{\frac{1-\lambda_{1} t^{2}}{1-t^{2}}}-\frac{1-\mathbf{E} / \mathbf{K}}{\sqrt{\left(1-t^{2}\right)\left(1-\lambda_{1} t^{2}\right)}}\right]=\frac{\mathcal{A}}{\pi \mathbf{K}}\left[\mathbf{E}^{\prime} \mathbf{K}+\mathbf{E K}^{\prime}-\mathbf{K} \mathbf{K}^{\prime}\right]=\frac{\mathcal{A}}{2 \mathbf{K}},
$$

where in the first step we used the integral expression for $\mathbf{E}^{\prime}=\mathbf{E}\left(\lambda_{1}\right)$ and $\mathbf{K}^{\prime}=\mathbf{K}\left(\lambda_{1}\right)$, and in the last step we have used Legendre's relation. Now $\mathcal{A}$ is fixed to

$$
\mathcal{A}=\frac{2 \mathbf{K}}{\pi} p_{F} \rightarrow\left\{\begin{array}{cc}
p_{F} & (\lambda \rightarrow 0) \\
\frac{p_{F}}{\pi} \ln \frac{16}{\lambda_{1}} & (\lambda \rightarrow 1)
\end{array}\right.
$$

Now the only remaining variational parameter is $\lambda$.

(ii) The spatially modulating part: Next, we treat the spatially modulating part (whose spatial average is zero). It is given by (see Fig. 3 for a reminder)

$$
-N \frac{\mathcal{A}^{2}}{2 \pi}\left(\int_{0}^{\omega_{F}}+\int_{-\omega_{F}}^{0}+\int_{-\omega_{\Lambda}}^{-\omega_{F}^{\prime}}\right) \mathrm{d} \omega \frac{\operatorname{sgn}\left(\omega^{2}-\mathcal{A}^{2} \mathbf{E} / \mathbf{K}\right)}{\sqrt{\left(\omega^{2}-\mathcal{A}^{2}\right)\left(\omega^{2}-\lambda_{1} \mathcal{A}^{2}\right)}} \mathcal{F}(\xi) .
$$

The integral part from the first energy branches in the Fermi (Dirac) sea gives

$$
-\int_{0}^{\omega_{F}} \frac{\mathrm{d} \omega}{\sqrt{\left(\omega^{2}-\mathcal{A}^{2}\right)\left(\omega^{2}-\lambda_{1} \mathcal{A}^{2}\right)}}=-\frac{1}{\mathcal{A}} \int_{0}^{1} \frac{\mathrm{d} t}{\sqrt{\left(1-t^{2}\right)\left(1-\lambda_{1} t^{2}\right)}}=-\frac{\mathbf{K}^{\prime}(\lambda)}{\mathcal{A}},
$$

where we have changed the variable as $\omega=\sqrt{\lambda_{1}} \mathcal{A} t$. On the other hand, the second energy branch in the Dirac sea gives a contribution with the same size but opposite $\operatorname{sign}\left(\omega_{F}^{\prime}=\mathcal{A}\right)$,

$$
\int_{-\omega_{\Lambda}}^{-\mathcal{A}} \frac{\mathrm{d} \omega}{\sqrt{\left(\omega^{2}-\mathcal{A}^{2}\right)\left(\omega^{2}-\lambda_{1} \mathcal{A}^{2}\right)}}=\frac{\mathbf{K}^{\prime}(\lambda)}{\mathcal{A}} .
$$

This can be checked by noting that the change of the variable $\omega \rightarrow 1 / \omega^{\prime}$ converts the integral into the same form as that in Eq.(57). Note that the Dirac sea contributions from the first and the second energy branches cancel out, leaving only the net contribution from the Fermi sea.

Assembling the spatial average and modulating parts, the fermion number density is given by

$$
\left\langle\psi^{\dagger} \psi(x)\right\rangle=N \frac{p_{F}}{\pi}\left(1+\frac{\mathbf{K K}^{\prime}}{\pi} \mathcal{F}(\xi)\right), \quad(\xi=\mathcal{A} x)
$$

where we have used $\mathcal{A}=2 p_{F} \mathbf{K} / \pi$ in Eq.(55). The behavior at $\lambda=0.9$ is plotted in Fig. 4 , 


\section{B. Energy density: Determination of $\lambda$}

Next we compute the energy density. The single-particle energy contribution gives

$$
\begin{aligned}
\mathcal{E}_{1}(x) & \equiv 2 N\left(\int_{0}^{\omega_{F}}+\int_{-\omega_{\Lambda}}^{0}\right) \frac{\mathrm{d} \omega}{2 \pi} \mathcal{D}(\omega) \omega\left(\left|\tilde{\varphi}_{\omega}(\xi)\right|^{2}+\left|\tilde{\chi}_{\omega}(\xi)\right|^{2}\right) \\
& =-N \int_{\omega_{F}^{\prime}}^{\omega_{\Lambda}} \frac{\mathrm{d} \omega}{2 \pi} \mathcal{D}(\omega) \omega\left[2-\frac{\mathcal{F}(\xi)}{\omega^{2} / \mathcal{A}^{2}-\mathbf{E} / \mathbf{K}}\right],
\end{aligned}
$$

where the integrand is an odd function of $\omega$, so that Fermi and Dirac sea contributions from the first energy branches cancel, leaving the contribution from the second energy branch in

the Dirac sea. (We have changed the variable as $\omega \rightarrow-\omega$.) Writing the spatial average and spatial fluctuation parts as $\overline{\mathcal{E}}_{1}$ and $\Delta \mathcal{E}$, straightforward calculations lead to

$$
\begin{aligned}
\frac{\overline{\mathcal{E}}_{1}^{R}}{N} & =-\frac{\mathcal{A}^{2}}{4 \pi}\left[\left(2-\lambda-2 \frac{\mathbf{E}}{\mathbf{K}}\right) \ln \frac{4 \Lambda^{2}}{\lambda \mathcal{A}^{2}}+\left(2-\lambda-4 \frac{\mathbf{E}}{\mathbf{K}}\right)\right], \\
\frac{\Delta \mathcal{E}_{1}}{N} & =\frac{\mathcal{A}^{2}}{4 \pi} \mathcal{F}(\xi) \ln \frac{4 \Lambda^{2}}{\lambda \mathcal{A}^{2}},
\end{aligned}
$$

where we define the regularized energy $\overline{\mathcal{E}}_{1}^{R} \equiv \overline{\mathcal{E}}_{1}-\mathcal{E}_{\text {vac }}$ where $\mathcal{E}_{\text {vac }}=-N \Lambda^{2} / 2 \pi$, and drop the $O(1 / \Lambda)$ terms.

Next we consider the contribution from the condensation terms. We first note that

$$
\frac{M^{2}(x)}{\mathcal{A}^{2}}=\lambda^{2} \frac{\operatorname{sn}^{2}(\xi) \mathrm{cn}^{2}(\xi)}{\operatorname{dn}^{2}(\xi)}=\left(2-\lambda-2 \frac{\mathbf{E}}{\mathbf{K}}\right)-\mathcal{F}(\xi),
$$

where the first bracket gives the spatial average, as we can see from the second bracket which is vanishing after averaging over the period $2 \mathbf{K}$. These terms have coefficient $1 / G$ whose renormalized value is determined through the renormalization condition,

$$
\frac{1}{G(\Lambda)}=\frac{1}{2 \pi} \ln \frac{4 \Lambda^{2}}{M_{0}^{2}}
$$

where $M_{0}$ is the effective mass in vacuum. With this expression, we have the average and fluctuation parts of the condensation energy $\left(\mathcal{E}_{2} / N \equiv M^{2}(x) / 2 G\right)$

$$
\frac{\overline{\mathcal{E}}_{2}}{N}=\frac{\mathcal{A}^{2}}{4 \pi}\left(2-\lambda-2 \frac{\mathbf{E}}{\mathbf{K}}\right) \ln \frac{4 \Lambda^{2}}{M_{0}^{2}}, \quad \frac{\Delta \mathcal{E}_{2}}{N}=-\frac{\mathcal{A}^{2}}{4 \pi} \mathcal{F}(\xi) \ln \frac{4 \Lambda^{2}}{M_{0}^{2}} .
$$

After combining $\mathcal{E}_{1}$ and $\mathcal{E}_{2}$, we can erase $\Lambda$ in the logarithms, and the energy depends on $\Lambda$ only through the renormalized paramemeter. Now we can write down the average and fluctuating parts of total energy. The average part is

$$
\frac{\left(\overline{\mathcal{E}}_{1}+\overline{\mathcal{E}}_{2}\right)_{R}}{N}=-\frac{\mathcal{A}^{2}}{4 \pi}\left[\left(2-\lambda-2 \frac{\mathbf{E}}{\mathbf{K}}\right) \ln \frac{M_{0}^{2}}{\lambda \mathcal{A}^{2}}+\left(2-\lambda-4 \frac{\mathbf{E}}{\mathbf{K}}\right)\right],
$$


where $\mathcal{A}=2 p_{F} \mathbf{K} / \pi$ due to the fermion number constraints; see Eq. (55). We have to choose the value of $\lambda$ so as to minimize the total average energy density. Using a relation

$$
\partial_{\lambda} \mathbf{E}=\partial_{\lambda} \int_{0}^{1} \mathrm{~d} t \sqrt{\frac{1-\lambda t^{2}}{1-t^{2}}}=\frac{\mathbf{E}-\mathbf{K}}{2 \lambda},
$$

we can show that only terms with the logarithmic coefficient survive:

$$
\partial_{\lambda}\left(\overline{\mathcal{E}}_{1}+\overline{\mathcal{E}}_{2}\right)=0 \quad \rightarrow \quad 0=\ln \frac{M_{0}^{2}}{\lambda \mathcal{A}^{2}} \times \partial_{\lambda}\left[\mathcal{A}^{2}\left(2-\lambda-2 \frac{\mathbf{E}}{\mathbf{K}}\right)\right]
$$

Thus we get a transcendental equation from the vanishing logarithmic term,

$$
M_{0}=\sqrt{\lambda} \mathcal{A}(\lambda)=\frac{2 p_{F}}{\pi} \times \sqrt{\lambda} \mathbf{K}(\lambda)
$$

which determines the optimal $\lambda$ as a function of $p_{F} / M_{0}$. Note that the optimized $\lambda$ makes the spatial modulating part of the energy density vanishing,

$$
\frac{\Delta\left(\mathcal{E}_{1}+\mathcal{E}_{2}\right)}{N}=\frac{\mathcal{A}^{2}}{4 \pi} \mathcal{F}(\xi) \ln \frac{M_{0}^{2}}{\lambda \mathcal{A}^{2}} \rightarrow 0 .
$$

meaning that the energy density is uniform everywhere.

\section{Scalar density: The self-consistency condition}

The scalar density can be expressed as

$$
\langle\bar{\psi} \psi(x)\rangle=-2 N\left(\int_{0}^{\omega_{F}}+\int_{-\omega_{F}}^{0}+\int_{-\omega_{\Lambda}}^{-\omega_{F}^{\prime}}\right) \frac{\mathrm{d} \omega}{2 \pi} \mathcal{D}(\omega)\left[\tilde{\varphi}_{\omega}^{*} \tilde{\chi}_{\omega}(\xi)+\tilde{\chi}_{\omega}^{*} \tilde{\varphi}_{\omega}(\xi)\right] .
$$

Note that in contrast to the fermion number density, the integrand is an odd function of $\omega$ because $\tilde{\varphi}_{\omega}=\tilde{\varphi}_{-\omega}$ and $\tilde{\chi}_{\omega}=-\tilde{\chi}_{-\omega}$. As a consequence, the contributions from the first energy branches in the Fermi and Dirac sea cancel, and only the third integral in 70 gives the net contribution. Therefore, in the GN model, the net contribution to the scalar density is dominated by the Dirac sea contribution. We will discuss this point in more detail in Sec.IV.

Changing the variable to keep the integration domain in positive values, we have

$$
\langle\bar{\psi} \psi(x)\rangle=2 N \int_{\omega_{F}^{\prime}}^{\omega_{\Lambda}} \frac{\mathrm{d} \omega}{2 \pi} \mathcal{D}(\omega)\left[\tilde{\varphi}_{\omega}^{*} \tilde{\chi}_{\omega}(\xi)+\tilde{\chi}_{\omega}^{*} \tilde{\varphi}_{\omega}(\xi)\right]
$$

We can express $\tilde{\chi}$ in terms of $\tilde{\varphi}$, and we arrive at

$$
\tilde{\varphi}_{\omega}^{*} \tilde{\chi}_{\omega}+\tilde{\varphi}_{\omega} \tilde{\chi}_{\omega}^{*}=-\frac{\mathcal{A}}{\omega}\left[\tilde{\varphi}_{\omega}^{*}\left(\partial_{\xi}+\mathcal{M}\right) \tilde{\varphi}_{\omega}+\tilde{\varphi}_{\omega}\left(\partial_{\xi}+\mathcal{M}\right) \tilde{\varphi}_{\omega}^{*}\right]=-\frac{\mathcal{A}}{\omega}\left(\partial_{\xi}+2 \mathcal{M}\right)\left|\tilde{\varphi}_{\omega}\right|^{2}
$$


Using Eq.(38), straightforward calculations lead to

$$
\tilde{\varphi}_{\omega}^{*} \tilde{\chi}_{\omega}+\tilde{\varphi}_{\omega} \tilde{\chi}_{\omega}^{*}=-\frac{\omega / \mathcal{A}}{\omega^{2} / \mathcal{A}^{2}-\mathbf{E} / \mathbf{K}} \mathcal{M}(\xi)
$$

Note that the scalar density at any energy level is proportional to $\mathcal{M}$. Finally, we sum over all the levels for the second energy branch in the Dirac sea,

$$
\langle\bar{\psi} \psi(x)\rangle=-2 N \mathcal{A} \mathcal{M}(\xi) \int_{\omega_{F}^{\prime}}^{\omega_{\Lambda}} \frac{\mathrm{d} \omega}{2 \pi} \frac{\omega}{\sqrt{\left(\omega^{2}-\mathcal{A}^{2}\right)\left(\omega^{2}-\lambda_{1} \mathcal{A}^{2}\right)}},
$$

which yields

$$
\langle\bar{\psi} \psi(x)\rangle=-N \frac{\mathcal{A M}(\xi)}{2 \pi} \ln \frac{4 \Lambda^{2}}{\lambda \mathcal{A}^{2}}=-N \frac{M(x)}{G} .
$$

Here we have used the relation determined by energy minimization, $\sqrt{\lambda} \mathcal{A}=M_{0}=2 \Lambda \mathrm{e}^{-\frac{\pi}{G}}$. The final expression proves the self-consistent condition. The behavior of the scalar density at $\lambda=0.9$ is plotted in Fig. 4 .

\section{Pseudoscalar density}

Next we will investigate the pseudoscalar density. At energy $\omega$, we have $\left(\gamma_{5}=-\gamma^{5}\right)$

$$
\bar{\psi}_{\omega} \mathrm{i} \gamma_{5} \psi_{\omega}=2 N\left(\left|\tilde{\varphi}_{\omega}\right|^{2}-\left|\tilde{\chi}_{\omega}\right|^{2}\right)
$$

Note that the integrand is an even function of $\omega$ in contrast to the scalar density case. We

did similar calculations for the spatially modulating part of the fermion number density, and found that the Dirac sea contributions in the first and second energy branches cancel out by themselves. The situation is similar here. The net contribution to pseudoscalar density comes only from the Fermi sea,

$$
\frac{\left\langle\bar{\psi} \mathrm{i} \gamma_{5} \psi(x)\right\rangle}{N}=\frac{\mathcal{A}^{2}}{2 \pi} \int_{0}^{\omega_{F}} \mathrm{~d} \omega \frac{\mathrm{dn}^{2}(\xi)-\operatorname{dn}^{2}(\xi-\mathbf{K})}{\sqrt{\left(\omega^{2}-\mathcal{A}^{2}\right)\left(\omega^{2}-\lambda_{1} \mathcal{A}^{2}\right)}}=\frac{\mathbf{K}^{\prime} \mathcal{A}}{2 \pi}\left[\operatorname{dn}^{2}(\xi)-\operatorname{dn}^{2}(\xi-\mathbf{K})\right],
$$

where we have used the spectral weights which we have computed for the fermion number [see Eq. (57)]. Now we have verified that the pseudoscalar condensate exists in the GN model at finite density, as stated in Introduction. While its spatial average is zero, it is locally nonzero in space.

Actually it is more instructive to express the pseudoscalar density in another way. Using the Dirac equation, we can derive a relation,

$$
\frac{\bar{\psi}_{\omega} \mathrm{i} \gamma_{5} \psi_{\omega}}{N}=2\left(\left|\tilde{\varphi}_{\omega}\right|^{2}-\left|\tilde{\chi}_{\omega}\right|^{2}\right)=\frac{1}{\tilde{\omega}} \partial_{\xi}\left(\tilde{\varphi}_{\omega} \tilde{\chi}_{\omega}^{*}+\tilde{\varphi}_{\omega}^{*} \tilde{\chi}_{\omega}\right)=\frac{1}{2 \tilde{\omega}} \frac{\partial_{\xi}\left(\bar{\psi}_{\omega} \psi_{\omega}\right)}{N} .
$$



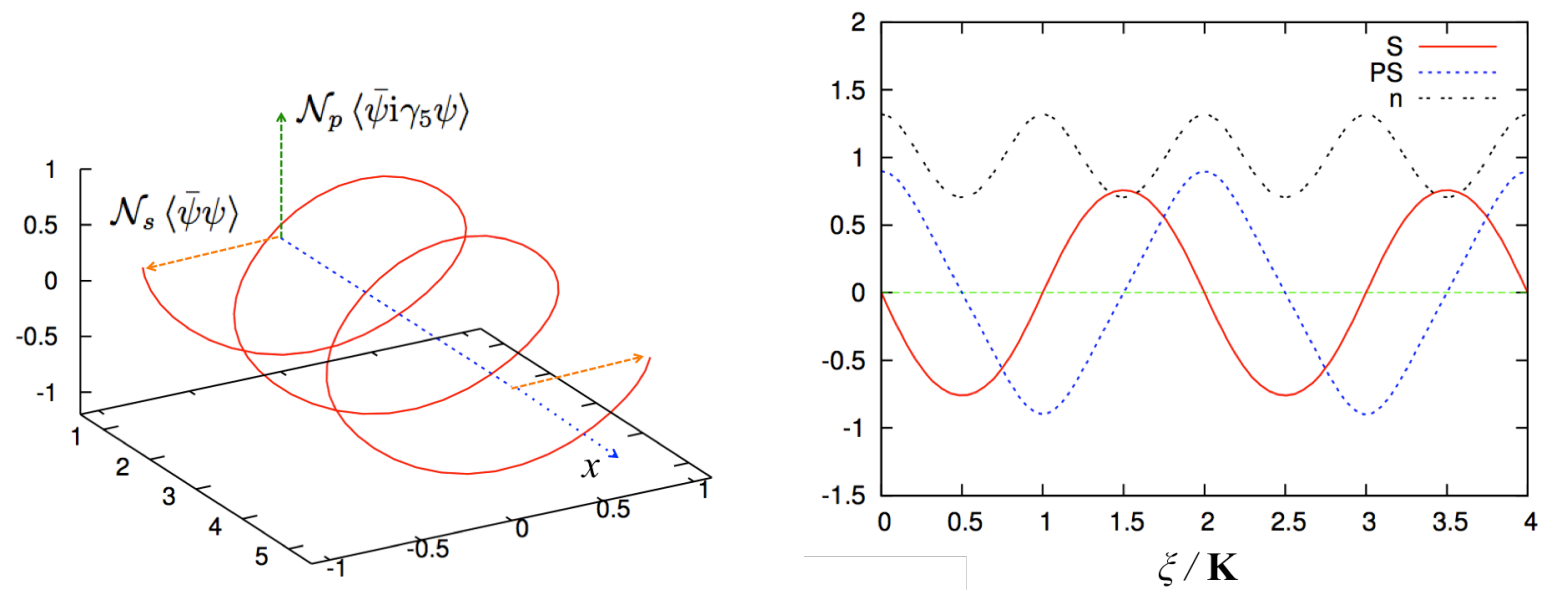

FIG. 4: Left: The "elliptic" chiral spirals at $\lambda=0.9$. We plot the amplitude-free parts of condensates, $\mathcal{N}_{s}\langle\bar{\psi} \psi\rangle \equiv\langle\bar{\psi} \psi\rangle \times G / N \mathcal{A}=-\mathcal{M}$ and $\mathcal{N}_{p}\left\langle\bar{\psi} \mathrm{i} \gamma_{5} \psi\right\rangle \equiv\left\langle\bar{\psi} \mathrm{i} \gamma_{5} \psi\right\rangle \times 2 \pi / N \mathbf{K}^{\prime} \mathcal{A}=\partial_{\xi} \mathcal{M}$. Right: The plots at $\lambda=0.9$ for the (normalized) scalar (S), pseudoscalar (PS), and fermion number density (n). For the fermion number density, we divide by $N p_{F} / \pi$. The fermion number is stuck at the location of the domain wall where the scalar density passes zero.

Therefore at given $\omega$, the pseudoscalar density is proportional to the spatial gradient of the scalar density. After integrating over $\omega$ with the spectral weight, we find

$$
\left\langle\bar{\psi} \mathrm{i} \gamma_{5} \psi(x)\right\rangle=N \frac{\mathbf{K}^{\prime} \mathcal{A}}{2 \pi} \partial_{\xi} \mathcal{M}(\xi) \text {. }
$$

This expression clarifies that the inhomogeneity of the chiral scalar condensate drives the formation of the pseudoscalar condensate. The typical behavior is shown in Fig. 4 .

\section{E. High and low density limits}

We consider the high and low density limits and examine qualitative aspects of the quantities which we have computed so far. To begin with, we first express $\lambda$ in terms of $p_{F} / M_{0}$. The transcendental equation (68) in the $\lambda \rightarrow 0$ and $\lambda \rightarrow 1$ cases becomes

$$
\frac{\pi M_{0}}{2 p_{F}}=\sqrt{\lambda} \mathbf{K}(\lambda) \rightarrow\left\{\begin{array}{cc}
\frac{\sqrt{\lambda} \pi}{2}\left[1+\frac{\lambda}{4}+O\left(\lambda^{2}\right)\right] & (\lambda \rightarrow 0) \\
\frac{1}{2} \ln \frac{16}{\lambda_{1}}+O\left(\lambda_{1} \ln \lambda_{1}\right) & (\lambda \rightarrow 1)
\end{array}\right.
$$

from which we get

$$
\lambda=\left\{\begin{array}{ll}
\left(\frac{M_{0}}{p_{F}}\right)^{2}-\frac{1}{2}\left(\frac{M_{0}}{p_{F}}\right)^{4}+O\left(M_{0}^{6} / p_{F}^{6}\right) & \left(M_{0} / p_{F} \ll 1\right) \\
1-16 \mathrm{e}^{-\pi M_{0} / p_{F}}+O\left(\mathrm{e}^{-2 \pi M_{0} / p_{F}}\right) & \left(1 \ll M_{0} / p_{F}\right)
\end{array} .\right.
$$


Next, we look at the parameter $\mathcal{A}$ which appears in place of the coordinate, $\xi=\mathcal{A} x$. Its asymptotic behavior is given by

$$
\mathcal{A}=\frac{2 \mathbf{K}(\lambda)}{\pi} p_{F}=\left\{\begin{array}{cl}
p_{F}\left[1+\frac{1}{4}\left(\frac{M_{0}}{p_{F}}\right)^{2}+O\left(M_{0}^{4} / p_{F}^{4}\right)\right] & \left(M_{0} / p_{F} \ll 1\right) \\
M_{0}+O\left(p_{F} \mathrm{e}^{-\pi M_{0} / p_{F}}\right) & \left(1 \ll M_{0} / p_{F}\right)
\end{array} .\right.
$$

Now we shall consider the physical quantities of particular interest.

(i) The asymptotic behavior of the energy gap is

$$
\Delta_{g} \equiv \omega_{F}^{\prime}-\omega_{F}=\left(1-\sqrt{\lambda_{1}}\right) \mathcal{A}=\left\{\begin{array}{cc}
M_{0} \times \frac{M_{0}}{2 p_{F}}+\cdots & \left(M_{0} / p_{F} \ll 1\right) \\
M_{0}+\cdots & \left(1 \ll M_{0} / p_{F}\right)
\end{array} .\right.
$$

In particular, at high density the gap is proportional to $1 / p_{F}$ and tends to close rather quickly. It is important to notice that this quick decreasing behavior is not generic in other two-dimensional models. For instance, in the $\mathrm{NJL}_{2}$ model the gap stays at the vacuum value, $\sim M_{0}$. We will discuss this issue more in Sec IV.

(ii) The low density behaviors of the chiral scalar and pseudoscalar condensates are

$$
\frac{\langle\bar{\psi} \psi\rangle}{N} \simeq-\frac{M_{0}}{G} \tanh \left(M_{0} x\right), \quad \frac{\left\langle\bar{\psi} \mathrm{i} \gamma_{5} \psi\right\rangle}{N} \simeq \frac{M_{0}}{4} \frac{1}{\cosh ^{2}\left(M_{0} x\right)} . \quad\left(1 \ll M_{0} / p_{F}\right)
$$

This is the solution for widely separated kinks. When the scalar density becomes zero, the pseudoscalar density is maximized. On the other hand, the scalar density is maximized when the pseudoscalar density is zero. Therefore, the combination of the scalar and pseudoscalar density forms the chiral spirals, as shown in Fig 4 .

(iii) The high density behaviors of the chiral scalar and pseudoscalar condensates are

$$
\frac{\langle\bar{\psi} \psi\rangle}{N} \simeq-\frac{\Delta_{g}}{G_{\Lambda}} \sin \left(2 p_{F} x\right), \quad \frac{\left\langle\bar{\psi} \mathrm{i} \gamma_{5} \psi\right\rangle}{N} \simeq \frac{\Delta_{g}}{G_{2 p_{F}}} \cos \left(2 p_{F} x\right), \quad\left(M_{0} / p_{F} \ll 1\right)
$$

where $G_{2 p_{F}}$ is defined by substituting $2 p_{F}$ in place of $\Lambda$ in the coupling constant $G(\Lambda)$. This disparity of the effective coupling constants reflects the fact that the scalar and pseudoscalar density acquire contributions from different domains. We can construct an approximate invariant,

$$
\left(G_{\Lambda}\langle\bar{\psi} \psi\rangle\right)^{2}+\left(G_{2 p_{F}}\left\langle\bar{\psi} \mathrm{i} \gamma_{5} \psi\right\rangle\right)^{2} \simeq N^{2} \Delta_{g}^{2} .
$$

As $p_{F}$ becomes larger, the expression approaches the chiral spirals with equal amplitudes for the scalar and pseudoscalar density. 

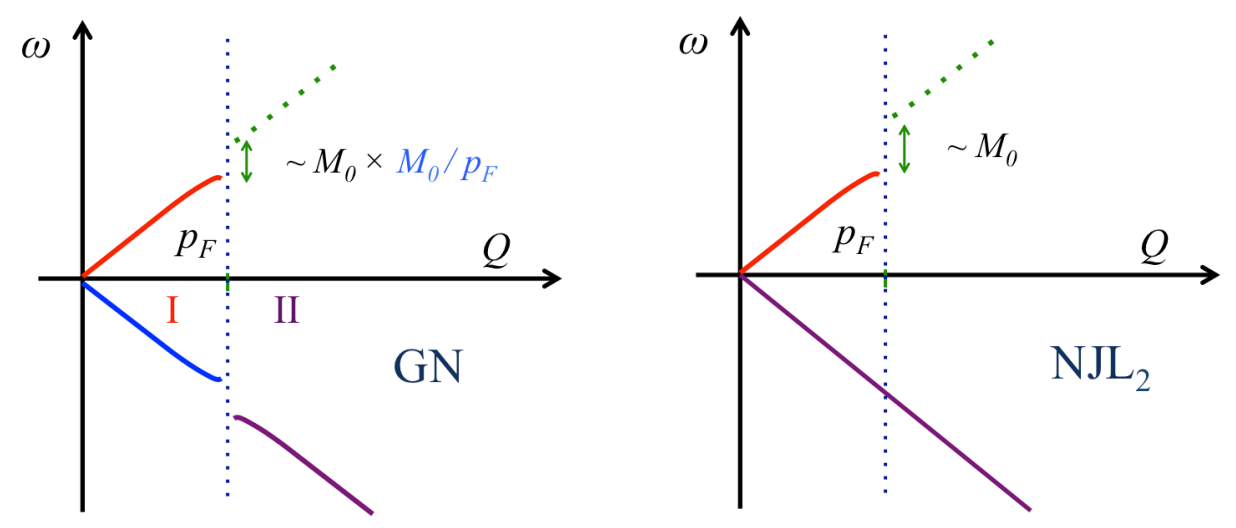

FIG. 5: The quasimomentum-energy dispersion. Left: For the GN model. The spectra contain the gapped region in the Fermi and Dirac sea. The size of the gap decreases like $\sim M_{0} \times M_{0} / p_{F}$ as $p_{F}$ becomes large. Right: For the $\mathrm{NJL}_{2}$-type models. The gaps open only at the Fermi points. The size of the gap is known to be $\sim M_{0}$, independently of the value of $p_{F}$.

\section{DISCUSSIONS}

In this section we examine the qualitative differences between the chiral spirals in the GN model and in the $\mathrm{QCD}_{2}$ or $\mathrm{NJL}_{2}$ models. First, we compare results of the GN model and of $\mathrm{QCD}_{2}$ by contrasting the short- and long-range interactions. Secondly, we argue why results of the GN and $\mathrm{NJL}_{2}$ models are qualitatively different, by emphasizing the structure of the 4-Fermi interactions.

\section{A. Short-range versus long-range interactions}

First let us recall the structure of the single-particle energy levels in the GN model. The energy level has gaps in the Fermi and Dirac seas; see Fig. 5. In the chiral scalar density, contributions from the first energy branch in the Fermi and Dirac sea cancel out, leaving only the contribution from the second energy branch in the Dirac sea [see Eq. (70)],

$$
\langle\bar{\psi} \psi\rangle_{\mathrm{I}}^{\text {Fermi }}+\left(\langle\bar{\psi} \psi\rangle_{\mathrm{I}}+\langle\bar{\psi} \psi\rangle_{\mathrm{II}}\right)^{\text {Dirac }}=\langle\bar{\psi} \psi\rangle_{\mathrm{II}}^{\text {Dirac }} . \quad(\mathrm{GN} \text { model })
$$

The condensate includes the contributions up to $\omega \sim-\Lambda$. This is the reason why the scalar density is proportional to $\sim \ln \left(2 \Lambda / M_{0}\right)$.

On the other hand, in the chiral pseudoscalar density, the Dirac sea contributions from 
the first and second energy branches cancel, leaving only the Fermi sea contribution,

$$
\left\langle\bar{\psi} \mathrm{i} \gamma_{5} \psi\right\rangle_{\mathrm{I}}^{\text {Fermi }}+\left(\left\langle\bar{\psi} \mathrm{i} \gamma_{5} \psi\right\rangle_{\mathrm{I}}+\left\langle\bar{\psi} \mathrm{i} \gamma_{5} \psi\right\rangle_{\mathrm{II}}\right)^{\text {Dirac }}=\left\langle\bar{\psi} \mathrm{i} \gamma_{5} \psi\right\rangle_{\mathrm{I}}^{\text {Fermi }} \quad(\mathrm{GN} \text { model }) .
$$

The amplitude is proportional to $\sim \ln \left(4 p_{F} / M_{0}\right)$. Due to the mismatch in the net contributions for the scalar and pseudoscalar density, their amplitudes are naturally different in the GN model.

When using this result as a guide for the QCD phase diagram, espcially when $p_{F}$ becomes larger than the vacuum quark effective mass, we should use the GN results with some caution. The above result strongly depends on the fact that the gaps in the Fermi and Dirac sea have the same size at the edge of the first energy branches. Such large gaps in the Dirac sea are rather specific to models with the contact interactions. In such models, although the condensation is initially driven by the low-energy particle-hole pairs near the Fermi surface, the created condensate affects spectra all the way from the Fermi surface down to the Dirac sea. Then the resulting gapped fermions in the Dirac sea also contribute to the condensate, giving large feedback to the fermions near the Fermi surface. Therefore there is a tight connection between the structures of the Fermi sea and Dirac sea.

In contrast, for models of the long-range interactions such as QCD, the physics near the Fermi surface does not strongly affect the structure of the Dirac sea. In fact, with momentum-dependent forces, the gap functions in general become momentum dependent.

If we had used models of long-range interactions such as $1 / \vec{p}^{2}$ force, the gap would be large near the Fermi surface but small otherwise. In particular, the chirality-violating effective mass tends to disappear in the Dirac sea as fermion density becomes large [6]. Then the main contribution to both chiral scalar and pseudoscalar density comes from the Fermi sea, and they tend to acquire the same amplitude. Actually, this is what happens in models like $\mathrm{QCD}_{2}$.

\section{B. The GN model versus the $\mathrm{NJL}_{2}$ model}

In the $\mathrm{NJL}_{2}$ model, the interaction is short range, as in the GN model. Nevertheless, qualtiative aspects of the chiral condensates are more similar to $\mathrm{QCD}_{2}$ rather than the GN model. Moreover, in contrast to the GN model, models in the latter class have an energy gap of $\sim M_{0}$ instead of a decreasing gap at finite density, $\sim M_{0}^{2} / p_{F}$ (Fig. 5). The key 
observation to understanding all these tendencies is that for a particular set of 4-Fermi interactions, physics near the Fermi surface tends to decouple from physics in the Dirac sea, as it happens for models with long-range interactions.

To explain this, first we project the fermion fields onto the right- and left-moving components,

$$
\psi_{r, l}=\frac{1 \pm \gamma_{0} \gamma_{z}}{2} \psi, \quad \gamma_{5}=\gamma_{0} \gamma_{z}
$$

For free fermions, the field equation is given by

$$
\left(p_{0}-p_{z}\right) \psi_{r}=0, \quad\left(p_{0}+p_{z}\right) \psi_{l}=0
$$

from which we observe that the right components have positive energy for $p_{z}>0$ and negative energy for $p_{z}<0$. The relation is opposite for the left components.

Now we express the 4-Fermi interactions in terms of left and right components. For bookkeeping purposes, let

$$
\Phi(x) \equiv \bar{\psi}_{l} \psi_{r}(x), \quad \Phi^{*}(x) \equiv \bar{\psi}_{r} \psi_{l}(x)
$$

The Fourier transform of $\Phi$ is $\left(\int_{x} \equiv \int \mathrm{d}^{2} x, \int_{q} \equiv \int \frac{\mathrm{d}^{2} q}{(2 \pi)^{2}}\right)$

$$
\Phi(q)=\int_{\delta p} \bar{\psi}_{l}\left(-\frac{q}{2}+\delta p\right) \psi_{r}\left(\frac{q}{2}+\delta p\right) .
$$

Notice that for $q_{z} \simeq 2 p_{F}$, both of the fields $\psi_{r}$ and $\psi_{l}$ at small $\delta p_{z}$ describe the fermion fields near the Fermi points. If $q_{z}$ is very different from $2 p_{F}$, either of the fields $\psi_{l}$ or $\psi_{r}$ in $\Phi(q)$ must be in the region far away from the Fermi surface, costing more energy. This is the reason why the homogeneous condensation at $q_{z}=0$ tends to disappear at finite density while instead the inhomogeneous condensate of $\left\langle\Phi\left(q_{z}=2 p_{F}\right)\right\rangle$ develops due to the condensed particle-hole pairs near the Fermi surface.

Now we consider the 4-Fermi interaction in the $\mathrm{NJL}_{2}$. It can be written as

$$
\int_{x}\left[(\bar{\psi} \psi)^{2}+\left(\bar{\psi} \mathrm{i} \gamma_{5} \psi\right)^{2}\right]=4 \int_{x}|\Phi(x)|^{2}=4 \int_{q} \Phi(q) \Phi^{*}(q),
$$

Note that the interaction couples $\Phi(q)$ with $\Phi^{*}(q)$. When one $\Phi(q)$ is replaced with the mean field $\left\langle\Phi\left(q_{z}=2 p_{F}\right)\right\rangle$, it affects only $\Phi^{*}\left(q_{z}=2 p_{F}\right)$ in which left- and right-moving fermions can simultaneously stay at low energy for small $\delta p_{z}$, and also simultaneously go to high energy for large $\delta p_{z}$. Phrasing this in another way, the mean field scatters low-energy fields 
to low energy, and high-energy fields to high energy, but it does not strongly mix up fields belonging to different energy domains.

The meaning of the above statements becomes clearer if we consider the GN model. Its 4 -Fermi interaction is

$$
\int_{x}(\bar{\psi} \psi)^{2}=\int_{x}\left[2|\Phi(x)|^{2}+\Phi^{2}+\Phi^{* 2}\right]=\int_{q}\left[2 \Phi(q) \Phi^{*}(q)+\Phi(q) \Phi(-q)+\Phi^{*}(q) \Phi^{*}(-q)\right],
$$

where we find extra couplings, $\Phi(q) \Phi(-q)$ and $\Phi^{*}(q) \Phi^{*}(-q)$. Now imagine that we have a mean field, $\left\langle\Phi\left(q_{z}=2 p_{F}\right)\right\rangle$. Then it couples to the composite field $\Phi\left(q_{z}=-2 p_{F}\right)$. But its content is

$$
\Phi\left(q_{z}=-2 p_{F}\right)=\int_{\delta p} \bar{\psi}_{l}\left(p_{F}+\delta p\right) \psi_{r}\left(-p_{F}+\delta p\right) .
$$

At small $\delta p_{z}$, both fields are in the Dirac sea according to the dispersion 90 . On the other hand, when the right field $\psi_{r}$ stays near the Fermi surface, then $\delta p_{z} \simeq 2 p_{F}$, and the $\psi_{l}$ field is in the Dirac sea with energy of $\sim-3 p_{F}$. Therefore, if the mean field is developed by condensation near the Fermi surface, it inevitably couples the fields near the Fermi surface to those in the Dirac sea. This is the reason why the GN model has the sizable energy gap not only near the Fermi surface but also in the Dirac sea. Furthermore, the feedback from the Dirac sea condensation strongly affects the Fermi surface condensations, making the inhomogeneous solutions far more complicated than those in the $\mathrm{NJL}_{2}$ model. Because of this feedback, the size of the mass gap in the GN model scales like $\sim M_{0}^{2} / p_{F}$, unlike $\sim M_{0}$ in the $\mathrm{NJL}_{2}$ model or $\mathrm{QCD}_{2}$.

Perhaps it is already clear why the results of chiral condensates in $\mathrm{NJL}_{2}$ and $\mathrm{QCD}_{2}$ are similar. In the former, the combinations of the 4-Fermi interactions are arranged in such a way that fields for the Fermi surface do not strongly couple to those for the Dirac sea. In the latter, the long-range interactions and the resulting momentum-dependent mass functions tend to forbid strong coupling between the Fermi surface and Dirac sea. In both cases, physics are governed by dynamics near the Fermi surface.

\section{SUMMARY}

In this paper, we have argued that the inhomogeneous chiral condensate in the GN model takes the chiral spiral form. Although the thermodynamic functional does not depend on 
the pseudoscalar density manifestly, the spatial modulations of the chiral scalar density inevitably generate the pseudoscalar density modulations.

While our arguments add little for the understanding of the GN model, the implications will become important once we start to infer proper effective models at finite quark density from more general perspectives on the fundamental theories. For assumed mean fields, we should calculate not only the thermodynamic functional but also various operators which might acquire large expectation values. Once such operators are found, we have to reanalyze the effective models including the interaction terms related to such operators, unless there is some reason to discard them. For the $\mathrm{NJL}_{4}$ up to dimensions-6 operators, it is perhaps

unavoidable to add $\left(\bar{\psi} \mathrm{i} \gamma_{0} \gamma_{j} \psi\right)^{2}$-type 4-Fermi interactions whose two-dimensional counterpart is $\left(\bar{\psi} \mathrm{i} \gamma_{5} \psi\right)_{2 \mathrm{D}}^{2}$. This is an approach pursued in Ref. [11].

We have also contrasted the GN model with the $\mathrm{NJL}_{2}$ model and $\mathrm{QCD}_{2}$ to understand the structure of the chiral spirals and the parametric behaviors of the mass gaps. In the GN model, the specific form of the 4-Fermi interaction and its short-range properties together create a strong Fermi-Dirac sea coupling which deforms the Dirac sea, even producing the energy gap inside the Dirac sea. If the interaction is replaced with the long-range one, such strong deformation of the Dirac sea tends to disappear. Similar results can be found if we arrange the 4-Fermi interactions in such a way as to weaken couplings between the Fermi and Dirac seas, as happened in the $\mathrm{NJL}_{2}$ model.

We found it very interesting that simple arrangements of the 4-Fermi interactions can control the coupling between the Fermi and Dirac sea contributions. Even within models with contact interactions, we can reproduce results similar to those in models with longrange interactions. Perhaps we may use the above kinematic considerations as a guide to restrict possible forms of the effective models at finite density, in addition to the ordinary symmetry considerations.

More implications from the GN model studies to the results of the $\mathrm{NJL}_{4}$ model will be discussed elsewhere.

\section{Acknowledgments}

T.K. acknowledges E. J. Ferrer, V. de la Incera, and S. Carignano for very stimulating discussions which motivate this work and for their kind hospitality during his visit to UTEP. 
This research was supported in part by NSF Grants No. PHY09-69790 and No. PHY1305891.

\section{Appendix A: Series expansion of the elliptic functions}

The series expansion for the elliptic functions is useful for several purposes, such as numerical computations. We have already given the expansion for Jacobi's $\theta$ function and $Z$ function in Eqs. 26) and (28). The expansions for the elliptic functions are $\left(q \equiv \mathrm{e}^{-\pi \mathbf{K}^{\prime} / \mathbf{K}}\right)$ 26 ]

$$
\begin{aligned}
& \operatorname{sn}(x \mid \lambda)=\frac{2 \pi}{\lambda^{1 / 2} \mathbf{K}} \sum_{n=0} \frac{q^{n+1 / 2}}{1-q^{2 n+1}} \sin \frac{(2 n+1) \pi x}{2 \mathbf{K}}, \\
& \operatorname{cn}(x \mid \lambda)=\frac{2 \pi}{\lambda^{1 / 2} \mathbf{K}} \sum_{n=0} \frac{q^{n+1 / 2}}{1+q^{2 n+1}} \cos \frac{(2 n+1) \pi x}{2 \mathbf{K}} \\
& \operatorname{dn}(x \mid \lambda)=-\frac{\pi}{2 \mathbf{K}}+\frac{2 \pi}{\mathbf{K}} \sum_{n=0} \frac{q^{n}}{1+q^{2 n}} \cos \frac{n \pi x}{\mathbf{K}} .
\end{aligned}
$$

For small $\lambda$, the elliptic functions can be expanded as [27]

$$
\operatorname{sn}(x \mid \lambda)=x-(1+\lambda) \frac{x^{3}}{3 !}+\cdots, \quad \operatorname{cn}(x \mid \lambda)=1-\frac{x^{2}}{2 !}+\cdots, \quad \operatorname{dn}(x \mid \lambda)=1-\lambda \frac{x^{2}}{2 !}+\cdots .
$$

\section{Appendix B: Relative phases}

To determine $\tilde{\chi}$ from $\tilde{\varphi}$ including the relative phase, we use Eqs.(6) and (35). We first compute the derivative. First we note that

$$
\frac{\mathrm{d} \tilde{\varphi}}{\mathrm{d} \xi}=\left[\frac{\pi}{2 \mathbf{K}}\left(\frac{1}{\theta_{1}\left(u_{\xi+\alpha}\right)} \frac{\mathrm{d} \theta_{1}\left(u_{\xi+\alpha}\right)}{\mathrm{d} u_{\xi+\alpha}}-\frac{1}{\theta_{4}\left(u_{\xi}\right)} \frac{\mathrm{d} \theta_{4}\left(u_{\xi}\right)}{\mathrm{d} u_{\xi}}\right)-Z(\alpha)\right] \tilde{\varphi}(\xi) .
$$

To proceed further, we need to use the formulas [28].

$$
\frac{1}{\theta_{1}\left(u_{a}\right)} \frac{\mathrm{d} \theta_{1}\left(u_{a}\right)}{\mathrm{d} u_{a}}=\frac{2 \mathbf{K}}{\pi}\left[Z(a)+\frac{\operatorname{cn}(a) \mathrm{dn}(a)}{\operatorname{sn}(a)}\right], \quad \frac{1}{\theta_{4}\left(u_{a}\right)} \frac{\mathrm{d} \theta_{4}\left(u_{a}\right)}{\mathrm{d} u_{a}}=\frac{2 \mathbf{K}}{\pi} Z(a),
$$

with which we get

$$
\frac{\mathrm{d} \tilde{\varphi}}{\mathrm{d} \xi}=\left[Z(\xi+\alpha)+\frac{\operatorname{cn}(\xi+\alpha) \operatorname{dn}(\xi+\alpha)}{\operatorname{sn}(\xi+\alpha)}-Z(\xi)-Z(\alpha)\right] \tilde{\varphi}(\xi) .
$$

Next we use the addition theorem for the zeta function [29],

$$
Z(\xi+\alpha)=Z(\xi)+Z(\alpha)-\lambda \operatorname{sn}(\xi) \operatorname{sn}(\alpha) \operatorname{sn}(\xi+\alpha)
$$


and for the elliptic functions [30],

$$
\begin{aligned}
\operatorname{sn}(u+v) & =\frac{\operatorname{sn}(u) \cdot \operatorname{cn}(v) \operatorname{dn}(v)+\operatorname{sn}(v) \cdot \operatorname{cn}(u) \operatorname{dn}(u)}{1-\lambda \operatorname{sn}^{2}(u) \cdot \operatorname{sn}^{2}(v)} \\
\operatorname{cn}(u+v) & =\frac{\operatorname{cn}(u) \cdot \operatorname{cn}(v)-\operatorname{sn}(u) \operatorname{dn}(u) \cdot \operatorname{sn}(v) \operatorname{dn}(v)}{1-\lambda \operatorname{sn}^{2}(u) \cdot \operatorname{sn}^{2}(v)} \\
\operatorname{dn}(u+v) & =\frac{\operatorname{dn}(u) \cdot \operatorname{dn}(v)-\lambda \operatorname{sn}(u) \operatorname{cn}(u) \cdot \operatorname{sn}(v) \operatorname{cn}(v)}{1-\lambda \operatorname{sn}^{2}(u) \cdot \operatorname{sn}^{2}(v)} .
\end{aligned}
$$

With these ingredients, we have to do messy calculations and get

$$
\tilde{\chi}_{\omega}(\xi)=-\operatorname{sgn}(\tilde{\omega}) \frac{1}{\operatorname{dn}(\xi)} \frac{\operatorname{cn}(\xi+\alpha)}{\operatorname{sn}(\xi+\alpha)} \tilde{\varphi}_{\omega}(\xi) .
$$

In the final step we use the relations among the theta functions and elliptic functions [Sec.(22.11) in Ref. [13]],

$$
\operatorname{sn}(a)=\frac{\theta_{3}(0)}{\theta_{2}(0)} \frac{\theta_{1}\left(u_{a}\right)}{\theta_{4}\left(u_{a}\right)}, \quad \operatorname{cn}(a)=\frac{\theta_{4}(0)}{\theta_{2}(0)} \frac{\theta_{2}\left(u_{a}\right)}{\theta_{4}\left(u_{a}\right)}, \quad \operatorname{dn}(a)=\frac{\theta_{4}(0)}{\theta_{3}(0)} \frac{\theta_{3}\left(u_{a}\right)}{\theta_{4}\left(u_{a}\right)},
$$

and then use the expression for $\tilde{\varphi}(\xi)$. The result is

$$
\tilde{\chi}_{\omega}(\xi)=-\operatorname{sgn}(\tilde{\omega}) \frac{\theta_{2}\left(u_{\xi+\alpha}\right)}{\theta_{3}\left(u_{\xi}\right)} \frac{\theta_{4}\left(u_{\xi}\right)}{\theta_{1}\left(u_{\xi+\alpha}\right)} \tilde{\varphi}_{\omega}(\xi)=-\mathcal{N} \operatorname{sgn}(\tilde{\omega}) \frac{\theta_{2}\left(u_{\xi+\alpha}\right)}{\theta_{3}\left(u_{\xi}\right)} \mathrm{e}^{\xi Z(\alpha)} .
$$

This expression can be converted into a more convenient form. To do this, we note that by definition the functions $\theta_{2}$ and $\theta_{3}$ are related to $\theta_{1}$ and $\theta_{4}$ as

$$
\theta_{2}(u)=\theta_{1}\left(u+u_{\mathbf{K}}\right)=-\theta_{1}\left(u-u_{\mathbf{K}}\right), \quad \theta_{3}(u)=\theta_{4}\left(u+u_{\mathbf{K}}\right)=\theta_{4}\left(u-u_{\mathbf{K}}\right),
$$

so the function $\tilde{\chi}(\xi)$ is proportional to $\tilde{\varphi}(\xi-\mathbf{K})$,

$$
\tilde{\chi}_{\omega}(\xi)=\operatorname{sgn}(\tilde{\omega}) \mathrm{e}^{\mathbf{K} Z(\alpha)} \times \mathcal{N} \frac{\theta_{1}\left(u_{\xi-\mathbf{K}+\alpha}\right)}{\theta_{4}\left(u_{\xi-\mathbf{K}}\right)} \mathrm{e}^{(\xi-\mathbf{K}) Z(\alpha)}=\operatorname{sgn}(\tilde{\omega}) \mathrm{e}^{\mathbf{K} Z(\alpha)} \times \tilde{\varphi}_{\omega}(\xi-\mathbf{K}),
$$

as it should be. Note that the exponent is purely imaginary.

\section{Appendix C: Normalization factor}

The equation to determine the normalization factor is

$$
\frac{1}{2}=\frac{1}{2 \mathbf{K}} \int_{0}^{2 \mathbf{K}} \mathrm{d} \xi\left|\tilde{\varphi}_{\omega}(\xi)\right|^{2}=\frac{\left|\mathcal{N}_{\omega}\right|^{2}}{2 \mathbf{K}} \int_{0}^{2 \mathbf{K}} \mathrm{d} \xi \frac{\theta_{1}\left(u_{\xi+\alpha}\right) \theta_{1}\left(u_{\xi+\alpha^{*}}\right)}{\theta_{4}^{2}\left(u_{\xi}\right)}
$$

for each energy $\omega$. We use the formula

$$
\theta_{3}^{2}(0) \theta_{1}(a+b) \theta_{1}(a-b)=\theta_{4}^{2}(a) \theta_{2}^{2}(b)-\theta_{4}^{2}(b) \theta_{2}^{2}(a)
$$


Similar formulas and derivations can be found in Chap. 21 in Ref. [13].

(i) The $\alpha=$ i $\eta$ case. In this case, $\alpha^{*}=-\alpha$, and we get

$$
\frac{\theta_{1}\left(u_{\xi+\alpha}\right) \theta_{1}\left(u_{\xi-\alpha}\right)}{\theta_{4}^{2}\left(u_{\xi}\right)}=\frac{\theta_{2}^{2}\left(u_{\alpha}\right)}{\theta_{3}^{2}(0)}\left[1-\frac{\theta_{4}^{2}\left(u_{\alpha}\right)}{\theta_{2}^{2}\left(u_{\alpha}\right)} \frac{\theta_{2}^{2}(\xi)}{\theta_{4}^{2}(\xi)}\right]=\frac{\theta_{2}^{2}\left(u_{\alpha}\right)}{\theta_{3}^{2}(0)}\left[1-\frac{\mathrm{cn}^{2}(\xi)}{\mathrm{cn}^{2}(\alpha)}\right],
$$

where we have used Eq. B7]. Finally, we take the spatial integral. Recalling Eq.(39), we find

$$
\left|\mathcal{N}_{\omega}\right|^{-2}=\frac{2}{\lambda \operatorname{cn}^{2}(\alpha)} \frac{\theta_{2}^{2}\left(u_{\alpha}\right)}{\theta_{3}^{2}(0)}\left[\operatorname{dn}^{2}(\alpha)-\frac{\mathbf{E}}{\mathbf{K}}\right] .
$$

Combining Eqs. C3) and (C4), we can arrive at the expression (38).

(ii) The $\alpha=\mathbf{K}+$ i $\eta$ case. We recall the relation (27), $\theta_{1}\left(u_{\xi+2 \mathbf{K}}\right)=-\theta_{1}\left(u_{\xi}\right)$. Then

$$
\theta_{1}\left(u_{\xi+\mathbf{K}+\mathrm{i} \eta}\right) \theta_{1}\left(u_{\xi+\mathbf{K}-\mathrm{i} \eta}\right)=-\theta_{1}\left(u_{\xi+\mathbf{K}+\mathrm{i} \eta}\right) \theta_{1}\left(u_{\xi-\mathbf{K}-\mathrm{i} \eta}\right)
$$

so $\theta_{1}\left(u_{\xi+\alpha}\right) \theta_{1}\left(u_{\xi+\alpha^{*}}\right)=-\theta_{1}\left(u_{\xi+\alpha}\right) \theta_{1}\left(u_{\xi-\alpha}\right)$. Therefore we can obtain results corresponding to Eqs. C3 and (C4) by multiplying them by $(-1)$. On the other hand, the $(-1)$ factors will cancel for the normalized probability function $|\tilde{\varphi}(\xi)|^{2}$, so Eq. (38) takes the same form for the $\alpha=\mathrm{i} \eta$ and $\mathbf{K}+\mathrm{i} \eta$ cases.

\section{Appendix D: Derivative of the Zeta-function}

To compute the derivative of the zeta function, we use the expression [31]

$$
Z(\alpha)=E(\alpha)-\alpha \mathbf{E} / \mathbf{K}
$$

where $E(\alpha)$ is the Jacobi incomplete elliptic integral,

$$
E(\alpha)=\int_{0}^{\alpha} \mathrm{d} w \mathrm{dn}^{2} w
$$

In particular, for $\alpha=\mathbf{K}$, we have the complete integral, $E(\mathbf{K})=\mathbf{E}$. From these expressions we can easily arrive at Eq.(42).

\section{Appendix E: More on the UV cutoff}

Here we will complete the discussions outlined in Sec.IID. First, we express $\omega_{\Lambda}$ by $\epsilon$. Using the Jacobi imaginary transformation and then applying the relation for the quarter period, we get $\left[\right.$ remember that $\left.\mathbf{K}^{\prime}(\lambda)=\mathbf{K}\left(\lambda_{1}\right)\right]$

$$
\frac{\omega_{\Lambda}}{\mathcal{A}}=\operatorname{dn}\left(\mathrm{i}\left(\mathbf{K}^{\prime}-\epsilon\right) \mid \lambda\right)=\frac{\operatorname{dn}\left(\mathbf{K}^{\prime}-\epsilon \mid \lambda_{1}\right)}{\operatorname{cn}\left(\mathbf{K}^{\prime}-\epsilon \mid \lambda_{1}\right)}=\frac{1}{\operatorname{sn}\left(\epsilon \mid \lambda_{1}\right)} .
$$


Then, expanding the elliptic functions via Eq.A2, we get

$$
\frac{\omega_{\Lambda}}{\mathcal{A}}=\frac{1}{\epsilon}\left[1+\left(1+\lambda_{1}\right) \frac{\epsilon^{2}}{3 !}+\cdots\right]
$$

Next, we treat our dispersion at quasimomenum $Q=\Lambda$,

$$
\frac{\Lambda}{\mathcal{A}}=-\mathrm{i} Z\left(\mathrm{i}\left(\mathbf{K}^{\prime}-\epsilon\right) \mid \lambda\right)+\frac{\pi}{2 \mathbf{K}} .
$$

Using the imaginary transformation formula [32],

$$
\mathrm{i} Z\left(\mathrm{i}\left(\mathbf{K}^{\prime}-\epsilon\right) \mid \lambda\right)=Z\left(\mathbf{K}^{\prime}-\epsilon \mid \lambda_{1}\right)-\operatorname{dn}\left(\mathbf{K}^{\prime}-\epsilon \mid \lambda_{1}\right) \frac{\operatorname{sn}\left(\mathbf{K}^{\prime}-\epsilon \mid \lambda_{1}\right)}{\operatorname{cn}\left(\mathbf{K}^{\prime}-\epsilon \mid \lambda_{1}\right)}+\frac{\pi}{2 \mathbf{K}}\left(1-\frac{\epsilon}{\mathbf{K}^{\prime}}\right) .
$$

The computation of the first term on the rhs requires comment. Using Eq.(D1) and then formulas [33], we get

$$
\begin{aligned}
Z\left(\mathbf{K}^{\prime}-\epsilon \mid \lambda_{1}\right) & =E\left(\mathbf{K}^{\prime}-\epsilon \mid \lambda_{1}\right)-\frac{\mathbf{E}^{\prime}}{\mathbf{K}^{\prime}}\left(\mathbf{K}^{\prime}-\epsilon\right) \\
& =\mathbf{E}^{\prime}-E\left(\epsilon \mid \lambda_{1}\right)+\lambda_{1} \frac{\operatorname{sn}\left(\epsilon \mid \lambda_{1}\right) \operatorname{cn}\left(\epsilon \mid \lambda_{1}\right)}{\operatorname{dn}\left(\epsilon \mid \lambda_{1}\right)}-\frac{\mathbf{E}^{\prime}}{\mathbf{K}^{\prime}}\left(\mathbf{K}^{\prime}-\epsilon\right) \\
& =-\epsilon\left(\lambda-\frac{\mathbf{E}^{\prime}}{\mathbf{K}^{\prime}}\right)+O\left(\epsilon^{3}\right) .
\end{aligned}
$$

The remaining calculations are straightforward. We can express the momentum cutoff $\Lambda$ as a function of $\epsilon$,

$$
\frac{\Lambda}{\mathcal{A}}=\frac{1}{\epsilon}+\epsilon\left[\frac{\lambda-2}{3}+\frac{\mathbf{E}}{\mathbf{K}}\right] .
$$

Combining Eqs. $(\mathrm{E} 2)$ and $(\mathrm{E} 6)$ to erase $\epsilon$, we arrive at Eq. (46) which expresses $\omega_{\Lambda}$ as a function of $\Lambda$.

\section{Appendix F: Location of the Fermi momentum}

In the main text, we have assumed that the location of the Fermi momentum coincides with the momentum at which the gaps open. In the following, we will verify this statement.

Suppose that the Fermi energy is larger than the energy where the gaps open. We write the Fermi energy as

$$
\epsilon_{F}=\omega_{F}^{\prime}+\delta \omega=\mathcal{A}+\delta \omega, \quad \delta \omega>0,
$$

where $\omega_{F}^{\prime}=\mathcal{A}$ is the minimum energy for the second energy branch. With nonzero $\delta \omega$, the energy density $\mathcal{E}$ is a function of $(\lambda, \mathcal{A}, \delta \omega)$. We will verify that the energy density increases for $\delta \omega>0$. 
First, we use the number density constraint to write $\mathcal{A}$ as a function of $\lambda$ and $\delta \omega$. Because the domain of the integration is changed by the existence of $\delta \omega$, we get the extra term in addition to terms we got before. It is given by $(\tilde{\omega}=\omega / \mathcal{A})$

$$
\int_{\mathcal{A}}^{\mathcal{A}+\delta \omega} \frac{\mathrm{d} \omega}{\pi} \frac{\tilde{\omega}^{2}-\mathbf{E} / \mathbf{K}}{\sqrt{\left(\tilde{\omega}^{2}-1\right)\left(\tilde{\omega}^{2}-\lambda_{1}\right)}} \simeq \frac{1}{\pi}\left(1-\frac{\mathbf{E}}{\mathbf{K}}\right) \sqrt{\frac{\mathcal{A} \delta \omega}{\lambda}} .
$$

Therefore, the number constraint is

$$
\frac{\mathcal{A}}{2 \mathbf{K}}+\frac{1}{\pi}\left(1-\frac{\mathbf{E}}{\mathbf{K}}\right) \sqrt{\frac{\mathcal{A} \delta \omega}{\lambda}}+O(\delta \omega)=\frac{p_{F}}{\pi} .
$$

This characterizes $\mathcal{A}$ as a function of $\lambda$ and $\delta \omega$, so $\mathcal{A}=\mathcal{A}(\lambda, \sqrt{\delta \omega})$. Now we expand $\mathcal{A}$ with respect to $\sqrt{\delta \omega}$, and write

$$
\frac{\mathcal{A}_{0}(\lambda)}{2 \mathbf{K}}=\frac{p_{F}}{\pi}, \quad \frac{\delta \mathcal{A}(\lambda, \sqrt{\delta \omega})}{2 \mathbf{K}}=-\frac{1}{\pi}\left(1-\frac{\mathbf{E}}{\mathbf{K}}\right) \sqrt{\frac{\mathcal{A}_{0}}{\lambda}} \sqrt{\delta \omega}<0 .
$$

Here we have used the fact $\lambda_{1} \leq \mathbf{E} / \mathbf{K} \leq 1$. With this expression, we can write the energy density as a function of two independent variables, $\mathcal{E}(\lambda, \sqrt{\delta \omega})$.

Like the computation for the number density, the change of integration domain adds an extra term to the energy density. It is given by

$$
\frac{\delta \mathcal{E}}{N}=\int_{\mathcal{A}}^{\mathcal{A}+\delta \omega} \frac{\mathrm{d} \omega}{\pi} \mathcal{D}(\omega) \omega \simeq \frac{\mathcal{A}}{\pi}\left(1-\frac{\mathbf{E}}{\mathbf{K}}\right) \sqrt{\frac{\mathcal{A} \delta \omega}{\lambda}} \simeq-\frac{\mathcal{A}_{0} \delta \mathcal{A}}{2 \mathbf{K}}+O(\delta \omega),
$$

where we have used Eq. (F4). Then the total energy density (homogeneous part) is

$$
\frac{\overline{\mathcal{E}}^{R}}{N} \simeq-\frac{\mathcal{A}^{2}}{4 \pi}\left[\left(2-\lambda-2 \frac{\mathbf{E}}{\mathbf{K}}\right) \ln \frac{M_{0}^{2}}{\lambda \mathcal{A}^{2}}+\left(2-\lambda-4 \frac{\mathbf{E}}{\mathbf{K}}\right)\right]-\frac{\mathcal{A}_{0} \delta \mathcal{A}}{2 \mathbf{K}} .
$$

Next, we expand the energy density around $\delta \omega=0$ and $\lambda=\lambda_{0}$ such that $M_{0}=\sqrt{\lambda_{0}} \mathcal{A}_{0}\left(\lambda_{0}\right)$. Because we verified that $\left.\partial_{\lambda} \mathcal{E}\right|_{\lambda=\lambda_{0}, \delta \omega=0}=0$ in Eq.(67), the correction of $\delta \lambda=\lambda-\lambda_{0}$ starts at the quadratic order. Therefore, the leading correction to $\mathcal{E}\left(\lambda_{0}, \delta \omega=0\right)$ starts with the $\sqrt{\delta \omega}$ term, and is given by

$$
\frac{\delta \mathcal{E}}{N}=\frac{\mathcal{A}_{0} \delta \mathcal{A}}{\pi}\left[\frac{\mathbf{E}}{\mathbf{K}}-\frac{\pi}{2}\right]>0 \quad(\delta \mathcal{A}<0, \quad \mathbf{E} / \mathbf{K}<\pi / 2) .
$$

This is the energy cost. Therefore, to minimize the energy, we must set $\delta \mathcal{A} \propto \sqrt{\delta \omega}$ to zero. We can repeat similar arguments for a Fermi momentum smaller than the momentum at the gapped point. This completes the proof.

[1] For early attempts, see D. V. Deryagin, D. Y. Grigoriev, and V. A. Rubakov, Int. J. Mod. Phys. A 7 (1992) 659; E. Shuster and D. T. Son, Nucl. Phys. B 573 (2000) 434 hep-ph/9905448; B. - 
Y. Park, M. Rho, A. Wirzba and I. Zahed, Phys. Rev. D 62 (2000) 034015 [hep-ph/9910347];

R. Rapp, E. V. Shuryak and I. Zahed, Phys. Rev. D 63 (2001) 034008 hep-ph/0008207.

[2] For review, M. Buballa and S. Carignano, arXiv:1406.1367 [hep-ph].

[3] D. Nickel, Phys. Rev. Lett. 103 (2009) 072301 [arXiv:0902.1778 [hep-ph]]; ibid. Phys. Rev. D 80 (2009) 074025 arXiv:0906.5295 [hep-ph]]; S. Carignano, D. Nickel and M. Buballa, Phys. Rev. D 82 (2010) 054009 [arXiv:1007.1397 [hep-ph]].

[4] H. Abuki, D. Ishibashi and K. Suzuki, Phys. Rev. D 85 (2012) 074002 arXiv:1109.1615 [hepph]]; E. Nakano and T. Tatsumi, Phys. Rev. D 71 (2005) 114006 [hep-ph/0411350].

[5] T. Kojo, Y. Hidaka, L. McLerran and R. D. Pisarski, Nucl. Phys. A 843 (2010) 37 arXiv:0912.3800 [hep-ph]].

[6] T. Kojo, Y. Hidaka, K. Fukushima, L. D. McLerran and R. D. Pisarski, Nucl. Phys. A 875 (2012) 94 arXiv:1107.2124 [hep-ph]]; T. Kojo, R. D. Pisarski and A. M. Tsvelik, Phys. Rev. D 82 (2010) 074015 arXiv:1007.0248 [hep-ph]].

[7] M. Thies, Phys. Rev. D 69 (2004) 067703 hep-th/0308164.

[8] M. Thies and K. Urlichs, Phys. Rev. D 67 (2003) 125015 hep-th/0302092.

[9] V. Schon and M. Thies, Phys. Rev. D 62 (2000) 096002 hep-th/0003195]; B. Bringoltz, Phys. Rev. D 79 (2009) 105021 arXiv:0811.4141 [hep-lat]]; ibid. 79 (2009) 125006 arXiv:0901.4035 [hep-lat]]; T. Kojo, Nucl. Phys. A 877 (2012) 70 arXiv:1106.2187 [hep-ph]].

[10] G. Basar, G. V. Dunne and M. Thies, Phys. Rev. D 79 (2009) 105012 arXiv:0903.1868 [hepth]].

[11] B. Feng, E. J. Ferrer and V. de la Incera, arXiv:1304.0256 [nucl-th].

[12] Handbook of Mathematical Functions, edited by M. Abramowitz and I. Stegun (Dover, New York, 1990).

[13] E. T. Whittaker and G. N. Watson, A Course of Modern Analysis, (Cambridge University Press, England, 1980).

[14] H. Li, D. Kusnezov and F. Iachello, J. Phys. A: Math. Gen. 33, 6413 (2000).

[15] G. V. Dunne and J. Feinberg, Phys. Rev. D 57 (1998) 1271 hep-th/9706012].

[16] The chiral spirals made of the chiral scalar and tensor condensates are dominated by pairs of particle-holes near the Fermi surface, and produce gaps. In contrast, the pionic chiral spirals in addition contain particle-antiparticle pairing, and have gaps in both the Fermi and Dirac seas. Discussions in Sec. IV] can be used to understand differences between these two types of 
chiral spirals.

[17] Formulas (16.13) and (16.15) in Ref. [12].

[18] Formulas (17.3.1) and (17.3.26) in Ref. [12].

[19] See also Table (16.8) in Ref. [12].

[20] See also the Table (16.16) in Ref. [12].

[21] Formula (16.27) in Ref. [12].

[22] Formula (17.4.38) in Ref. [12].

[23] When $\eta$ exceeds $\mathbf{K}^{\prime}, q \mathrm{e}^{\pi \eta / \mathbf{K}}=\mathrm{e}^{\pi\left(-\mathbf{K}^{\prime}+\eta\right) / \mathbf{K}}>1$, and power series in Eq.28 blow up. Thus, $\eta \in\left[0, \mathbf{K}^{\prime}\right]$.

[24] Formula (16.20) in Ref. [12].

[25] Formulas (17.2), (17.3) and the figure (17.2) in Ref. [12].

[26] Formula (16.23) in Ref. [12].

[27] Formula (16.22) in Ref. [12].

[28] Formula (16.34) in Ref. [12].

[29] Formula (17.4.35) in Ref. [12].

[30] Formula (16.17) in Ref. [12].

[31] Formula (17.4.28) in Ref. [12].

[32] Formula (17.4.36) in Ref. [12].

[33] Formulas (17.4.28) and (17.4.7) in Ref. [12]. 